\title{
Communication technique sur les ouvrages du Rhin
}

\author{
A technical paper on works \\ and structures of the Rhine
}

\author{
PAR R. LEFOULON, \\ CONTHÒlEUR GÉNÉRAL a L'ÉLECTRICITÉ DE FRANCE
}

\begin{abstract}
Depuis quinze ans, l'aménagentent du Rinin entre Bâle et Strasbourg se poursuit en même temps que l'aménagement du Rhône ì l'aval de Valence, équipements du même ordre de grandeur et également réatisés au double titre de la production de l'énergie et de la navigation.

Les mêmes problèmes hydrauliques ont rẹ̣l des solutions variantes sur les deux fleuves.

Après l'exposé très intéressant des aspects rencontrés sur le Rhône, il apparait utile d'éyoquer $\dot{a}$ titre de complément la conception el les méthodes de réalisation des principatx: ouvrages: barrage-bloc usine-déchargeurs-écluses, des biefs du Rhin, compte tenu des condifions particulières suivantes :

- nature du sol des fondations constituées par la masse alluvionnaire très profonde du sillon alsacien;

- maintien d'un trafic très important sur le fleuve pendant les travaux;

- dimensions imposées pour les oubrages de navigation;

- réalisation d'aménagements en chaine sur une même dérivation.

Tous les ouvrages ont fait l'objet d'études hydrauliques nombreuses et approfondies sur modèle réduit au Laboratoire de Chatou.
\end{abstract}

\section{Monsieur le Président, \\ Messieurs,}

A la suite de la très intéressante conférence de M. Gemaehling sur les aménagements du ticrs central du Rhône, il m'a paru intéressant de vous donner parallèlement quelques indications sur les problèmes analogues que nous avons été amenés à résoudre sur le Rhin pendant ces quinze dernières années.

Nos problèmes se sont différenciés de ceux du Rhône pour les raisons suivantes :
The development of the Rhine between nasle and Strasbourg has been running concurrently with that of the Rhone below Valence for fifteen years. Both programmes have involved similar-sized structures and have aimed simultaneously at the production of hydro-power and the development of river navigation. Similar problems on both rivers have been solved in different ways.

It seems only reasonable to follow up the highly interesting account of the various aspects of the problem on the Rhone with a description of the design and construction methods along each stretch of the Rhine, the dam, and the unit formed by the pouer house, relief works and locks, with due consideration to the following factors:

- The nature of the foundation soil, consisting of the very deen allubial mass of the Alsatian trollgh:

- The continued occurrence of nery heavy river traffic during the work;

- The dimensions laid down for the navigational structures:

- The construction of structures "in series" along a given diversion.

A considerable number of comprehensive hydranlic scale model tests were carried out at the Chatou Laboratory for all these structures.

1. Nature des fondations : alors que, sur le Rhône, le sol dur et imperméable était à faible distance du lit du fleuve, nous nous sommes trouvés, dans la Plaine d'Alsace, en présence d'une masse alluvionnaire très profonde (parfois plus de $100 \mathrm{~m}$ );

2. Les ouvrages de navigation avaient les dimensions imposées par la Commission Centrale pour la Navigation du Rhin;

3. Les ouvrages devaient être réalisés et mis en service sans gêne et a fortiori sans arrêt 
d'une navigation intense atteignant plus de 7 millions de tonnes par an;

4. Enfin, la réalisation des usines en série sur un canal latéral nous avait obligés, dès le début, à une conception de déchargeurs capables d'un fonctionnement de longue durée sans érosion des ouvrages.

Ma communication a essentiellement pour but de montrer que les études sur modèle réduit ont été fondamentales pour résoudre nos problèmes. Les perfectionnements apportés par les laboratoires d'Hydraulique pour réaliser une concordance toujours plus parfaite entre le modèle et la nature nous ont permis d'atteindre plus sûremenl nos buts.

Nous traiterons successivement de la conception, de la construction des barrages, des ouvrages de navigation, en particulier des écluses et des blocs usines-déchargeurs.

\section{I. - BARRAGES}

\section{A - Barrage de Marckolsheim.}

La Convention franco-allemande de 1956 nous a amenés à abandonner à la moitié de sa réalisation le projet de 1925 qui prévoyait la construction de huit chutes en série sur un canal latéral, parallèle au Rhin, depuis Bâle jusqu'à Strasbourg.

Après la quatrième chute, nous avons pris l'engagement de réaliser les chutes aval en dérivation sur le fleuve. Il s'agissaif de dispositions qui conduisaient à construire entre Brisach et Strasbourg quatre barrages sur le fleuve.

Les deux barrages précédemment construits sur cette partie du Rhin supérieur : Kembs, à l'aval de Bâle, en 1928-1932 et Birsfelden en 1954 à l'amont de Bâle, l'avaient été dans des conditions plus favorables que celles que nous tronvions à l'aval de Brisach quant à la nature des fondations et à l'importance de la navigation sur le fleuve.

D'une part, les deux barrages précités avaient pu être fondés sur une marne résistante et imperméable située à quelques mètres sous les alluvions; d'autre part, le trafic de la navigation n'avait été que de $300000 \mathrm{t}$ par an au moment de la construction de Kembs, il n'avait pas dépassé $700000 \mathrm{t}$ à l'amont de Bâle à la construction de Birsfelden.

Les quatre barrages à construire à l'aval de Vogelgrun devaient être, eux, fondés sur des alluvions très profondes.

Bien que nous ayons trouvé, pour le premier barrage de Marckolsheim, un site favorable, ò la profondeur des alluvions était de $19 \mathrm{~m}$, fonder l'ouvrage à cette profondeur aurait coûté extrêmement cher. Aussi étions-nous dans l'obligation de fonder le barrage sur l'alluvion, l'intérêt du site permettant de réaliser une coupure jusqu'au rocher à l'amont des fondations du barrage, par un rideau d'injections.

La construction devait se realiser sans gêne pour la navigation qui empruntait un chenal de $75 \mathrm{~m}$ situé près de la rive gauche.

Le trafic sur le fleuve, réalisé en grande par- tie par des automoteurs allant jusqu'à $2000 t$ et des convois tirés par de puissants remorqueurs rhénans de $4000 \mathrm{ch}$, a atteint pendant la période de construction 7 millions de tonnes par an avec un passage journalier à travers le chantier atteignant 130 bateaux.

Le barrage a été conçu pour évacuer un débit de $4800 \mathrm{~m}^{3} / \mathrm{s}$ avec une passe de réserve en vue de parer à une indisponibilité pour incident ou entretien. Ce débit de $4800 \mathrm{~m}^{3} / \mathrm{s}$ correspond à la crue millénaire de $6000 \mathrm{~m}^{3} / \mathrm{s}$, dont $1200 \mathrm{~m}^{3} / \mathrm{s}$ sont évacués par le canal de dérivation, même en cas d'indisponibilité des groupes de l'usine, par l'utilisation des déchargeurs.

Pour diminuer le plus possible le nombre de piles en rivières, qui devaient être obligatoirement construrtes par fonçage à air comprimé, la solution optimale consistait à construire cinq passes de $30 \mathrm{~m}$ de largeur et, par suite, quatre piles en rivière.

Une premier modèle, à deux dimensions dans un canal vitré, nous a permis d'étudier le meilleur profil à donner aux piles et aux radiers et de définir les protections nécessaires pour éviter les affouillements à proximité de l'ouvrage.

A titre indicatif, signalons les dimensions déjà importantes des caissons qui ont servi à réaliser les piles : $30,50 \mathrm{~m}$ de longueur, $10 \mathrm{~m}$ de largeur el $4,50 \mathrm{~m}$ de hauteur, où un personnel assez réduit travaillait à l'aise grâce à deux petits bulldozers.

Pour la protection des piles, les revêtements traditionnels en granit ont été remplacés par de la tôle d'acier de $16 \mathrm{~mm}$ qui servait de coffrage.

Dans la masse des alluvions, les radiers ont été bétonnés à l'abri de rideaux de palplanches en deux temps :

- il a d'abord été exécuté, sur une hauteur de $4,50 \mathrm{~m}$, un bétonnage sous l'eau;

- ensuite - au-dessits et à sec - un béton armé d'une hauteur variable de 4,50 à $1,50 \mathrm{~m}$, la liaison entre les deux parties étant réalisée par des éléments de profilés et de palplanches servant à la fois d'armature et d'échafaudage. 


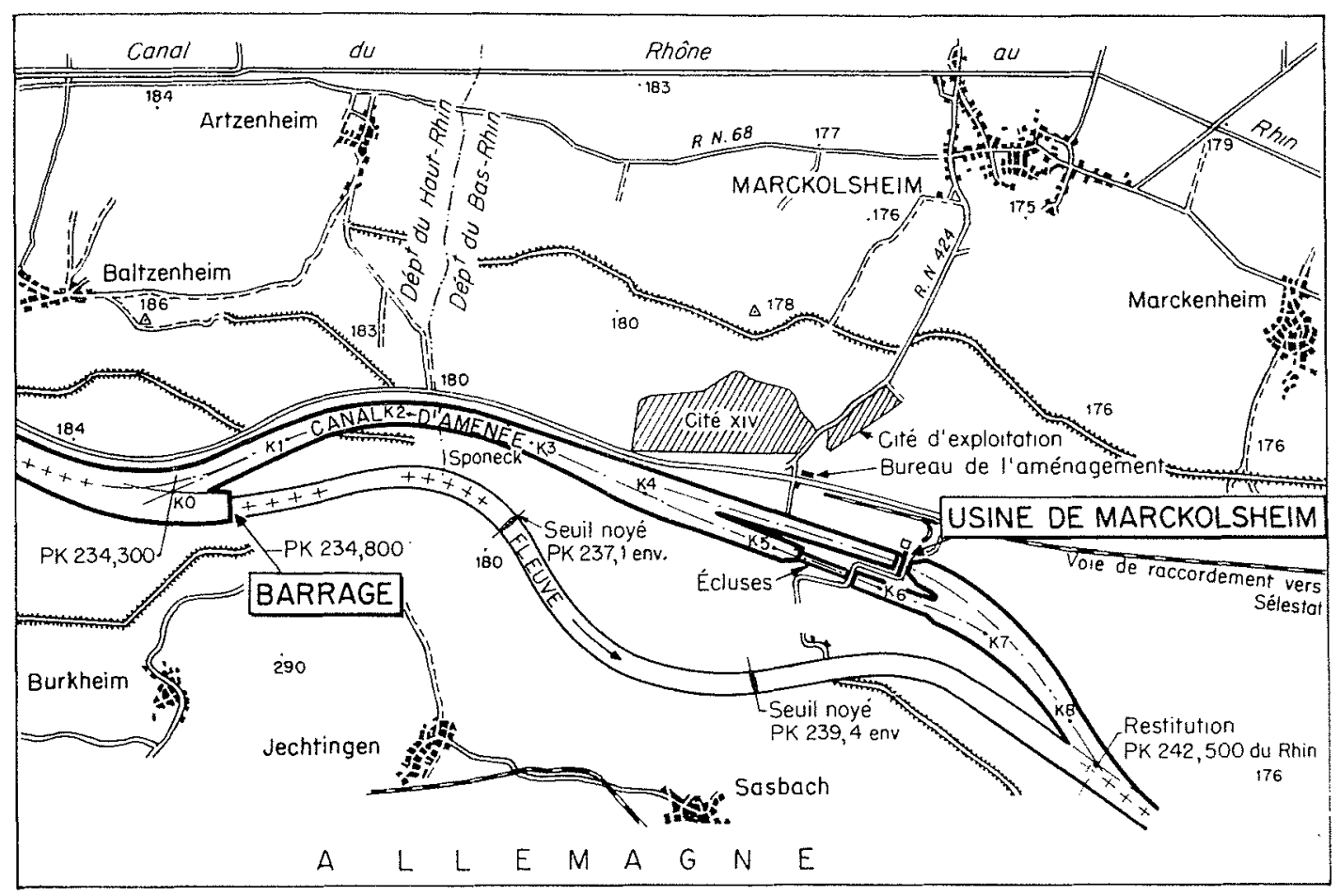

FIG. 1

Marckolsheim. Plan général.

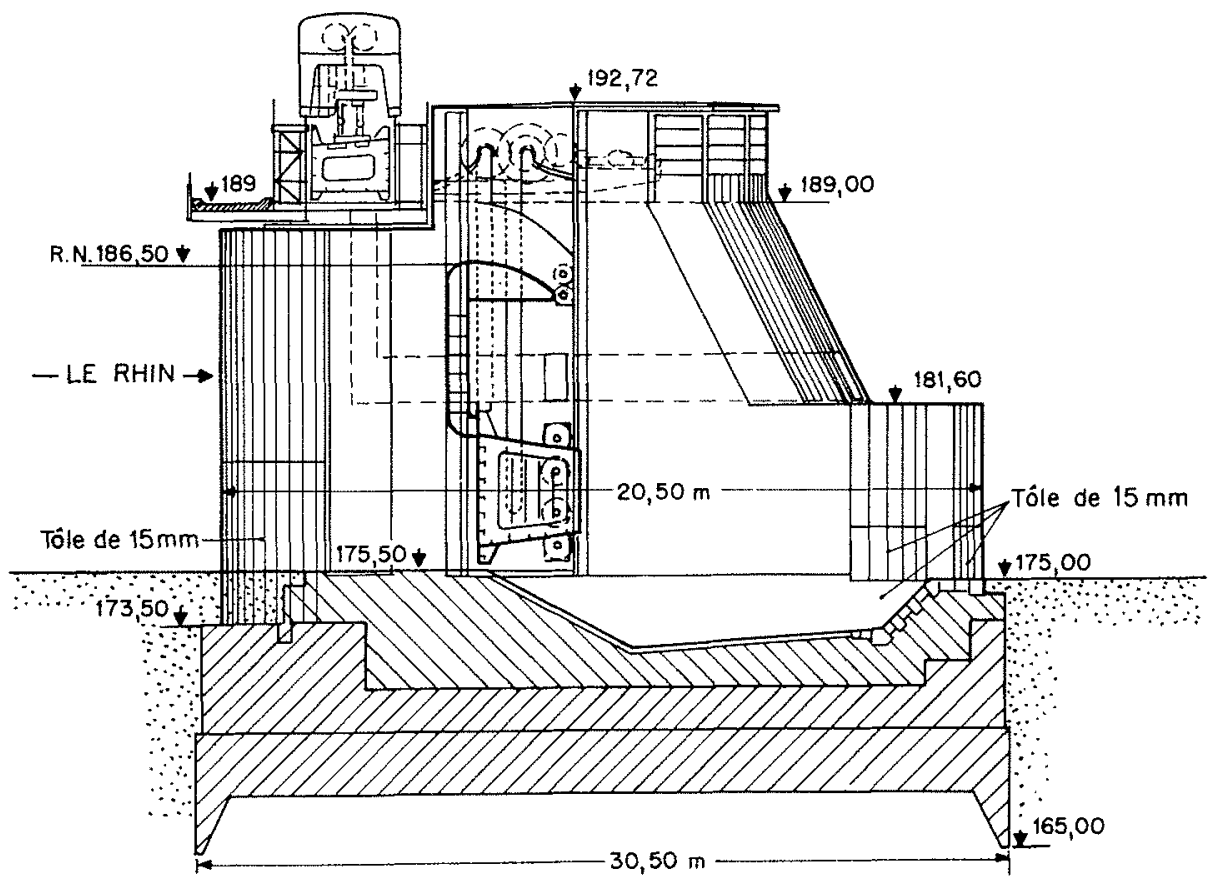

Fra. 2

Marckolsheim. Elévation d'une pile du barrage. 
Pour étudier les différentes phases de travaux dans le cours du Rhin à fond mobile et pour assurer un passage à la navigation avec le moins de gêne possible, en même temps qu'un débouché suffisant au passage des grandes crues, il a été décidé de faire construire par le Laboratoire de Chatou un modèle à trois dimensions à fond mobile, à grande échelle : $6 \mathrm{~km}$ de Rhin ont été reconstitués à l'échelle du $1 / 66^{\mathrm{e}}$. Le modèle occupait $100 \mathrm{~m}$ de longueur.

Les premiers essais ont eu pour but de définir les dimensions d'une dérivation provisoire sur la rive droite pour réduire le débit dans le chenal navigable pendant les périodes de hautes eaux tout en maintenant la totalité du débit dans le chenal pendant les périodes de basses eaux. Cette dérivation a permis de dériver de 10 à $20 \%$ du débit du fleuve.

Le modèle a été ensuite utilisé pour déterminer dans les différentes phases des travaux les vitesses dans la passe navigable, les pentes du fleuve, les courants traversiers, les efforts de traction, l'importance des affouillements et des dépôts consécutifs aux crues et aux décrues et la nécessité éventuelle de dragages. Il a permis également d'étudier les formes et dimensions des ouvrages : prises d'eau, musoirs, culées, et la protection des berges à l'amont et à l'aval de l'ouvrage.

Pour fixer les consignes de navigation, un bateau-modèle téléguidé de $1,56 \mathrm{~m}$ de long et de $17 \mathrm{~cm}$ de large a été utilisé.

Les phases des travaux sont indiquées dans les figures ci-dessous : elles montrent que nous avons maintenu pendant leur durée la navigation dans son chenal, le réduisant pendant la troisième phase à $60 \mathrm{~m}$ et en imposant l'alternat.

Pour illustrer la qualité des études sur modèle, je vous donnerai trois exemples de concordance nature-modèle pour les débits, les vitesses et les pentes superficielles.

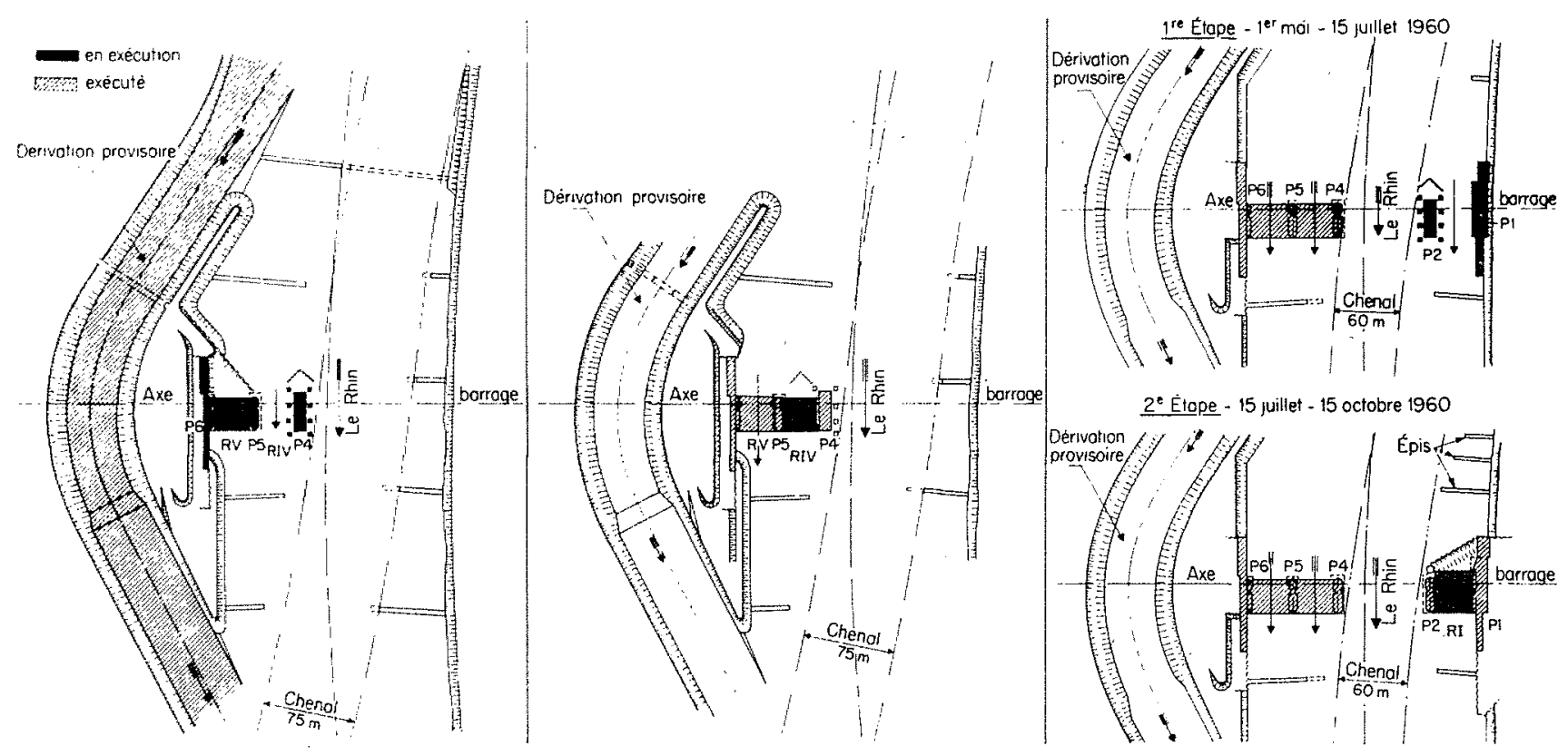

Fig. 3

Marckolsheim. Phases des travaux I, II, III.

Ire PHASE

Du $1^{\text {er }}$ octobre 1958 au $1^{\text {er }}$ janvier 1960. Durée : 15 mois.

Travaux du barrage :

- Génie civil : dérivation provisoire; culée $P 6$, pile $P 5$, pile P 4, radier $\mathrm{RV}$;

- Equipement : néant.

Navigation: chenal de $75 \mathrm{~m}$, sans changement;

Crue: débouché $140 \mathrm{~m}$.

\section{PHASE}

Du $1^{\text {er }}$ janvier au $1^{\text {er }}$ mai 1960 : 4 mois.

Travaux du barrage :

- Génie civil : radier 4 ;

- Equipement : néant.

Navigation: chenal de $75 \mathrm{~m}$, sans changement.

Crue: débouché de $130 \mathrm{~m}$.

\section{III ${ }^{3}$ PHASE}

Du $1^{\text {er }}$ mai au 15 octobre 1960: 5 mois $1 / 2$.

Travaux du barrage :

- Génie civil : piles 1 et 2;

- Equipement : vannes V et IV.

Navigation: chenal de $60 \mathrm{~m}$.

Crue: débouché de $120 \mathrm{~m}$. 
Fig. 4

Marckolsheim

Phase III.

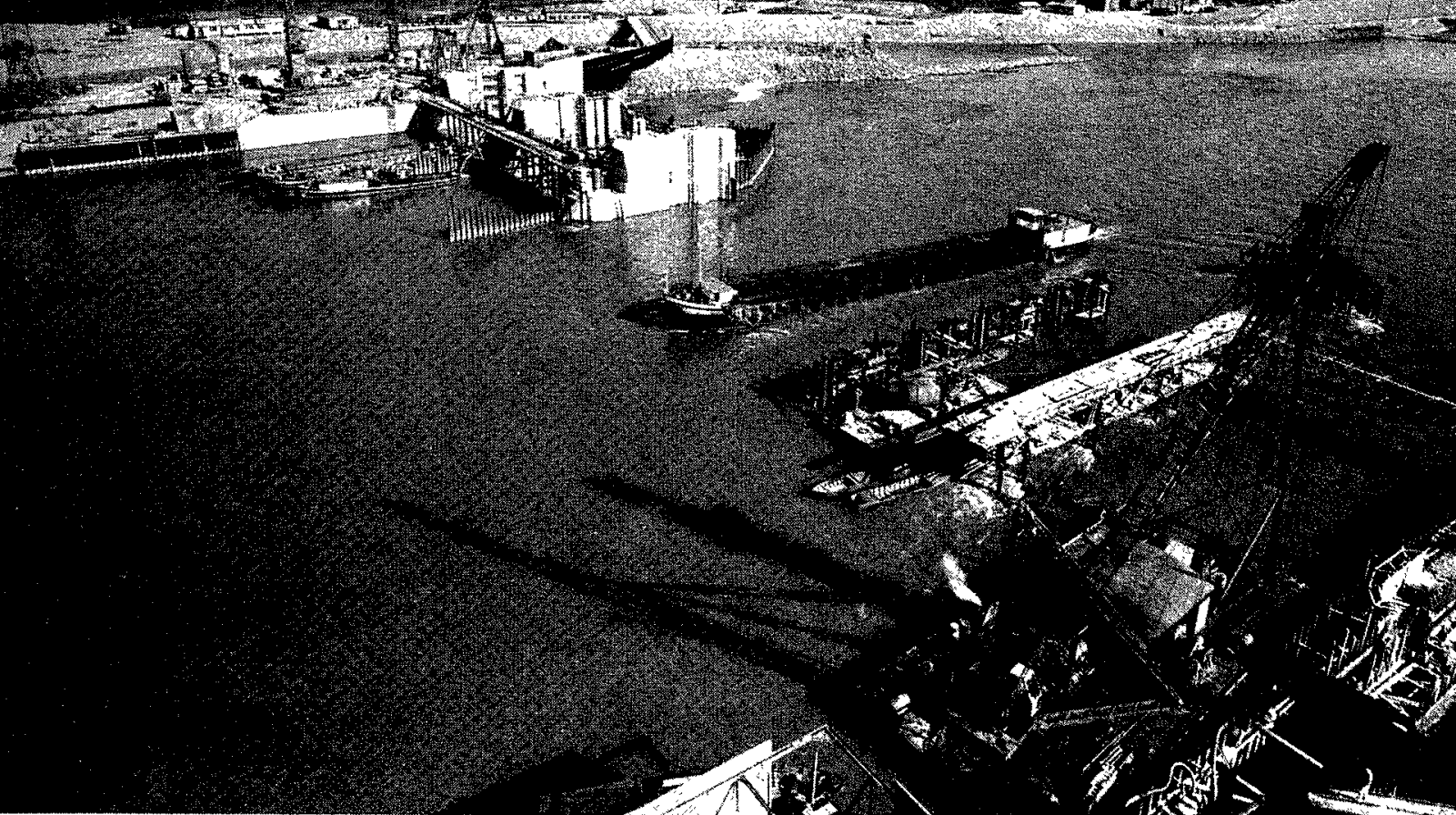

(Photo Baranger, Paris.)

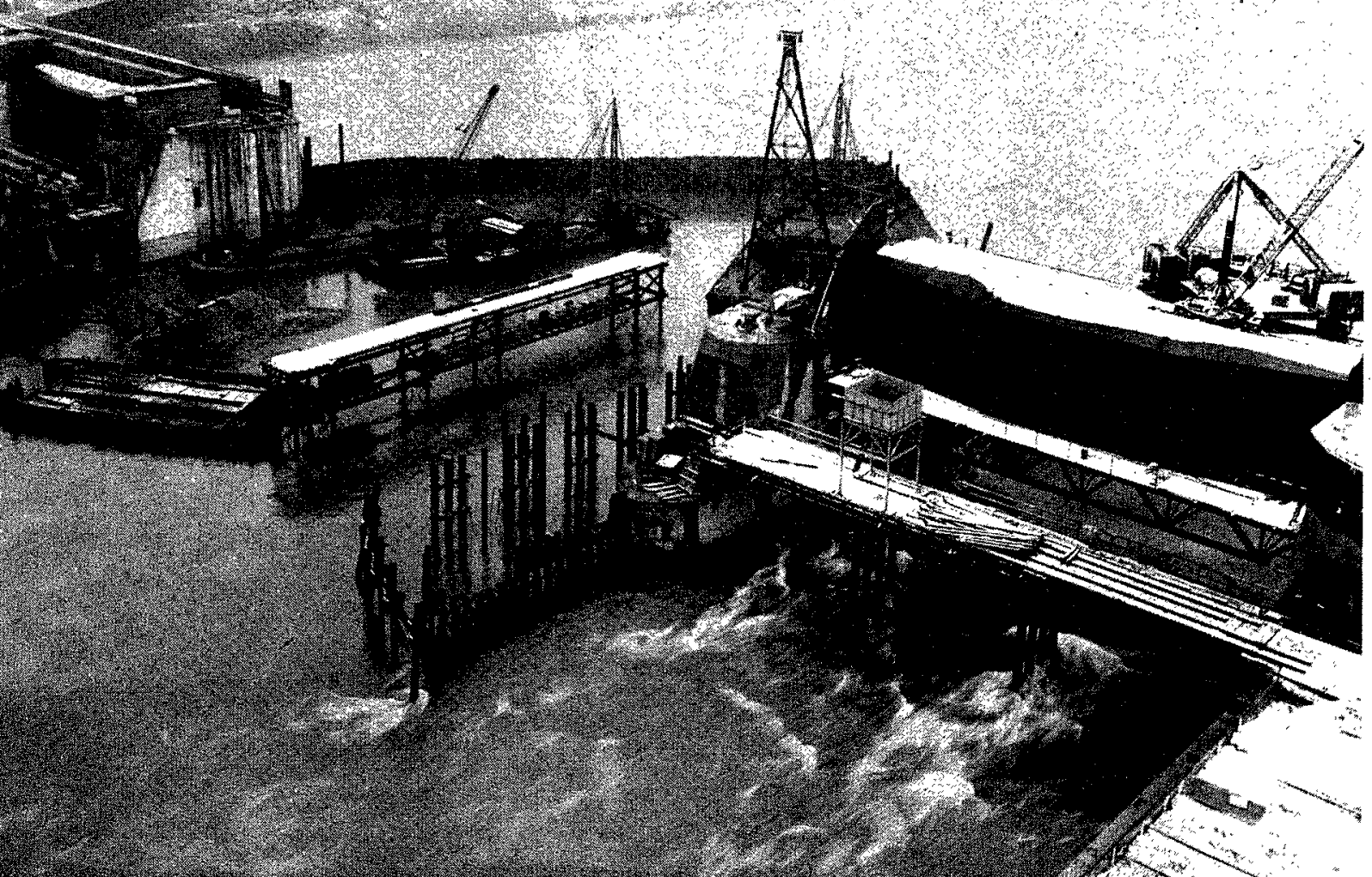

Fig. 5

Marckolsheim.

Phase IV. 


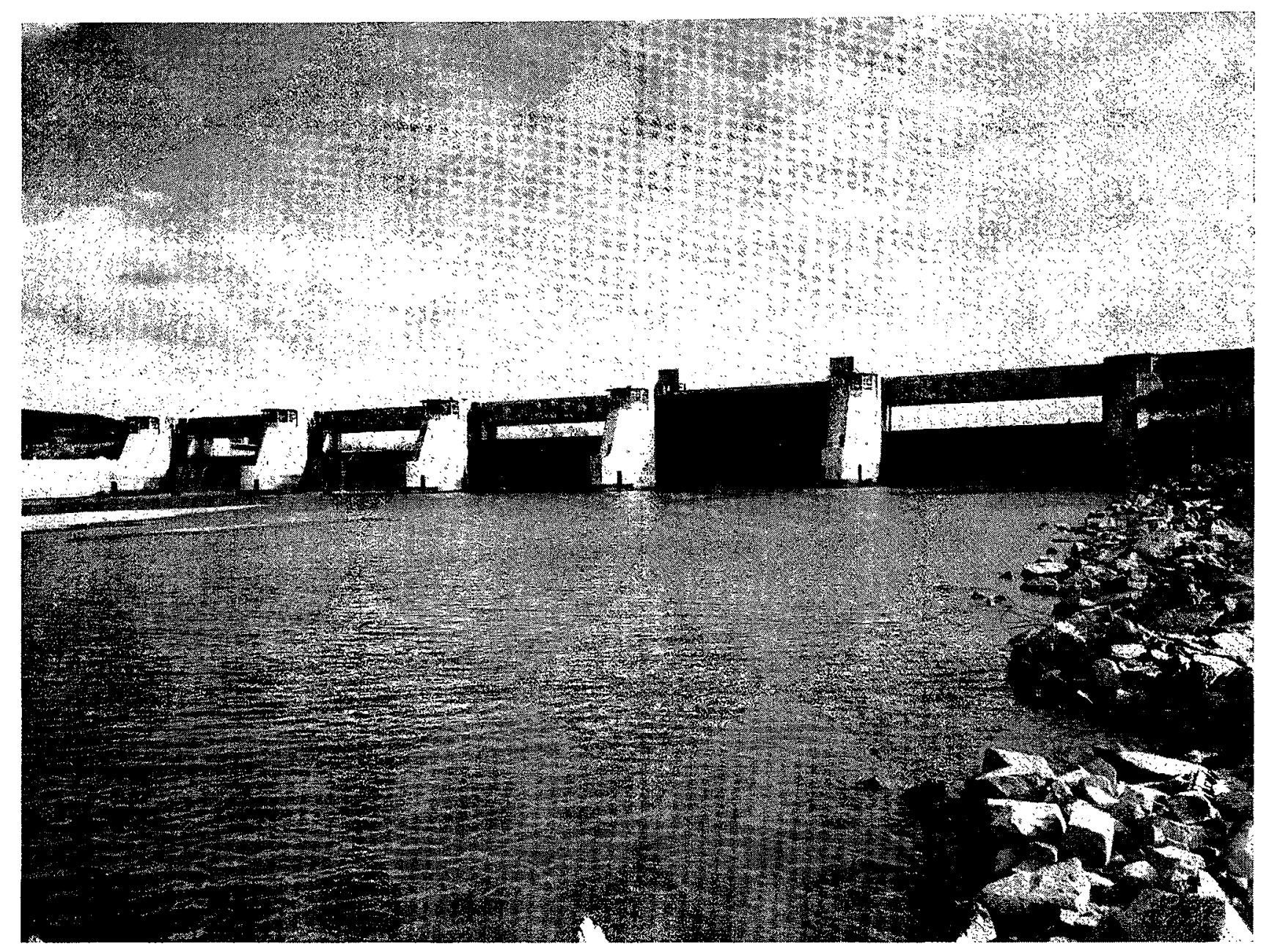

Fig. 6

(Photo Buranger', I'aris.)

Marckolsheim. Le barrage.

\section{CONCORvance DES DÉBITS :}

Les jaugeages effectués dans la dérivation provisoire ont permis d'apprécier la valeur du modèle. Le tableau suivant permet de comparer le pourcentage du débit total dérivé à celui mesuré sur modèle.

L'écart sensible sur les deux premiers jaugea- ges est dû à la surélévation à $178 \mathrm{du}$ seuil d'entrée de la dérivation provisoire, décidée pendant l'exécution des travaux des deux premières phases, de manière à avoir une profondeur d'eau suffisante. Avec un seuil à 177 de la cote d'entrée (celui du troisième jaugeage) on retrouve la parfaite concordance entre les débits dérivés nature et modèle.

\begin{tabular}{|c|c|c|c|c|}
\hline Date du JaUgeatie & $Q$ total & $Q$ dérivé & $\frac{Q d}{\mathrm{Q} t}$ & $\frac{\mathrm{Q} d}{\mathrm{Q} t}$ modèle \\
\hline & & & \\
\hline & $\left(\mathrm{m}^{3} / \mathrm{s}\right)$ & $\left(\mathrm{m}^{3} / \mathrm{s}\right)$ & $(\%)$ & $(\%)$ \\
$25 / 6 / 1959$ & 1200 & 40 & 3,3 & 10,4 \\
$8 / 7 / 1959$ & 1400 & 122 & 8,7 & 11,7 \\
$9 / 8 / 1960$ & 2000 & 329 & 16,5 & 15 \\
\hline
\end{tabular}




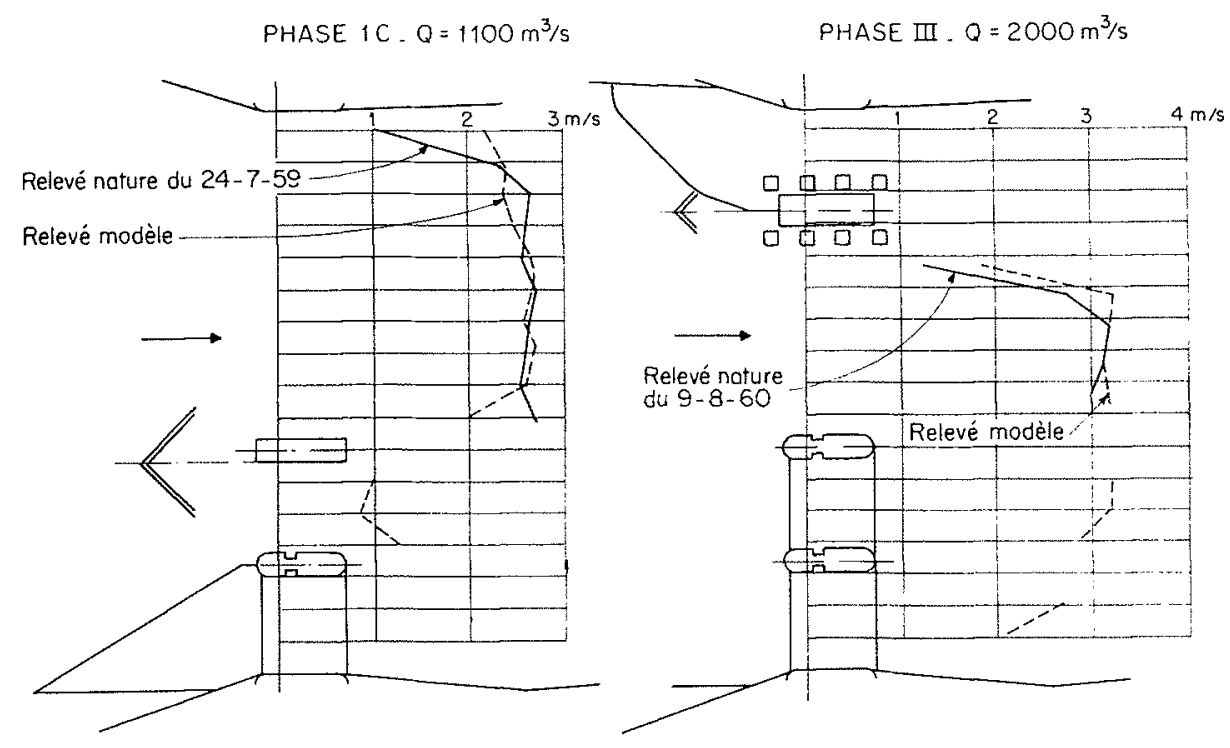

FIG. 7

Marckolsheim.

Diagramme de concordance des vitesses entre nature et modèle.

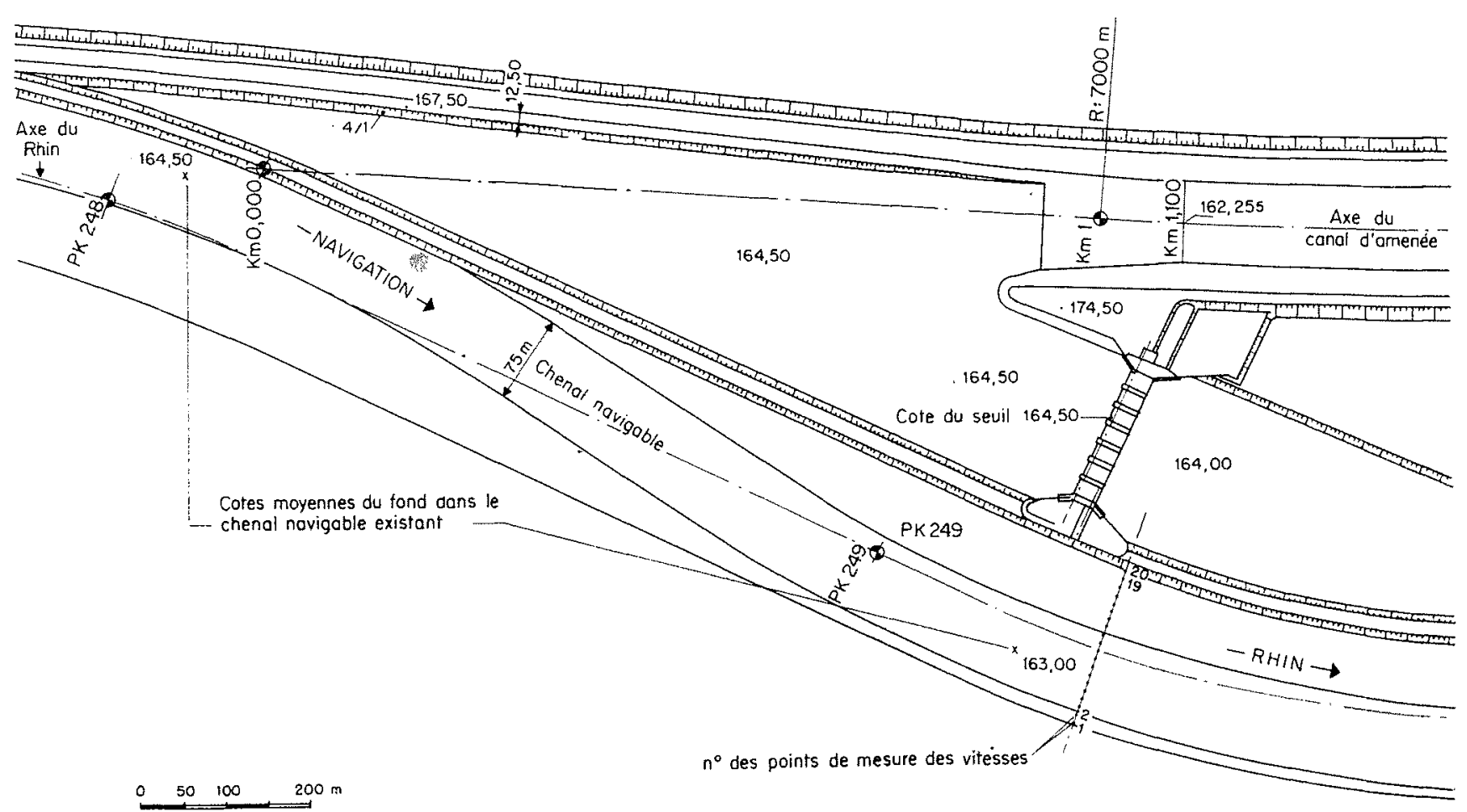

FIG. 8

Rhinau.

Plan général du barrage et de la prise.

Situation au jour 《J». 


\section{CONCORDANCE DES vitesses DANS LE RHIN:}

Sur la première partie de la figure 7 sont comparées les vitesses au cours de la phase I C : construction de la pile 4 et du radier 5 , avec un débit de $1100 \mathrm{~m}^{3} / \mathrm{s}$ dans le Rhin.

Sur la deuxième partie, ce sont les vitesses au cours de la phase III : construction de la pile 2 et du radier 1 , pour un débit de $2000 \mathrm{~m}^{3} / \mathrm{s}$.

Les écarts entre relevés nature et modèle sont inférieurs à $10 \%$, c'est-à-dire compris dans la double marge des erreurs de mesure et le plus souvent inférieurs à $5 \%$.

\section{Concondance des PENTES SUPERFICIElles DANS LA ZONE DU BARRAGE :}

Les essais sur modèle avaient montré la nécessité de réaliser trois épis sur la rive gauche à l'amont du barrage pendant la phase III : construction de la pile 2 et du radier 1 , de manière à ramener la pente de $3 \%$ à $2,4 \%$.

Dans le tableau ci-dessous sont indiquées les pentes maximales mesurées sur $70 \mathrm{~m}$ de longueur dans la nature et sur modèle, précisant que pour les mesures sur le chantier on a utilisé un remorqueur immobilisé dans le courant, dont on relevait les positions successives:

- en plan, par intersection des visées de deux théodolites placés, l'un sur la pile 4, l'autre sur la rive droite du Rhin à $200 \mathrm{~m}$ à l'amont ou à l'aval du barrage;

- en altitude, par lecture du niveau sur une mire placée à la verticale du centre de flottaison.

\begin{tabular}{|c|c|c|}
\hline & $\begin{array}{c}\text { Nature } \\
(\%)\end{array}$ & $\begin{array}{c}\text { Modèle } \\
(\%)\end{array}$ \\
\hline Premier essai ....... & 1,8 & 1,9 \\
Deuxième essai....... & 1,8 & 1,9 \\
Troisième essai ..... & 2,4 & 2,2 \\
\hline
\end{tabular}

\section{B - Conception du barrage de Rhinau.}

A la fin de l'année 1958, nous mettions au point les phases de construction du barrage de Marckolsheim et nous avions conscience des difficultés de la construction de ce barrage en rivière.

A la même époque, nous étudiions le projet de l'aménagement de Rhinau et, pour nous libérer des sujétions des travaux dans le lit du fleuve et de la présence de la navigation, nous avons conçu la construction à sec d'un barrage mobile implanté sur la rive gauche du fleuve et prolongé par une digue en alluvions dans le lit du fleuve. Pendant la construction du barrage mobile le fleuve serait maintenu dans son lit et la navigation continuerait à être pratiquée normalement; par ailleurs, l'écoulement des crues était inchangé. Après la construction du barrage mobile et $d u$ canal de dérivation, ces deux ouvrages seraient mis en eau. Par l'ouverture d'un bouchon, la navigation serait déviée dans le canal et on procéderait à la fermeture du lit du Rhin par une digue en alluvions, en utilisant concurremment et progressivement pour l'écoulement du fleuve les vannages du barrage mobile et le canal de dérivation.

La possibilité de néaliser le barrage à sec dans la masse des alluvions, à l'abri d'un simple batardeau en palplanches, permettait de reconsidérer la conception de l'ouvrage.

C'est ainsi qu'après examen de diverses solutions, nous nous sommes arrêtés à un barrage comprenant sept passes de $20 \mathrm{~m}$ de largeur dont une de réserve, obturée par des vannes-segment très classiques, de $20 \mathrm{~m}$ de largeur et de $9,50 \mathrm{~m}$ de hauteur, avec clapet déversant de $2,50 \mathrm{~m}$.

Le projet définitivement arrêté fut présenté à l'approbation des Commissions Internationales en février 1959 et l'étude sur un modèle réduit au $1 / 66^{\mathrm{e}}$ au Laboratoire de Chatou a permis de fixer les formes du radier, la protection à réaliser à l'amont et à l'aval du barrage.

Actuellement, le barrage est presque achevé; sa mise en eau interviendra au milieu de l'année 1963.

C'est également sur le modèle qu'ont été mis au point les trois phases principales de la mise en eau, qui pourra se réaliser même si survient une crue de $2000 \mathrm{~m}^{3} / \mathrm{s}$. Il a été en outre vérifié que la crue décennale de $3500 \mathrm{~m}^{3} / \mathrm{s}$ n'aurait pas de conséquence néfaste sur les ouvrages pendant cette mise en eau :

1. Ouverture partielle du bouchon pour une passe navigable de $130 \mathrm{~m}$ à l'entrée de la dérivation.

Le barrage mobile est maintenu fermé et, avec les déblais du bouchon, on commence la digue de fermeture du Rhin sur une longueur de 50 à $60 \mathrm{~m}$.

$2^{\circ}$ La navigation, qui a été maintenue dans le Rhin pendant la première phase, est alors dérivée dans le canal et en huit jour's doit se réaliser la coupure du Rhin, le débit du fleuve étant évacué progressivement par le barrage mobile.

$3^{\circ}$ La troisième phase comporte l'achèvement de la digue de fermeture et l'ouverture complète des bouchons. 
Fig. 9

Rhinau.

Vue aérienne sur barrage et entrée du canal d'amenée.

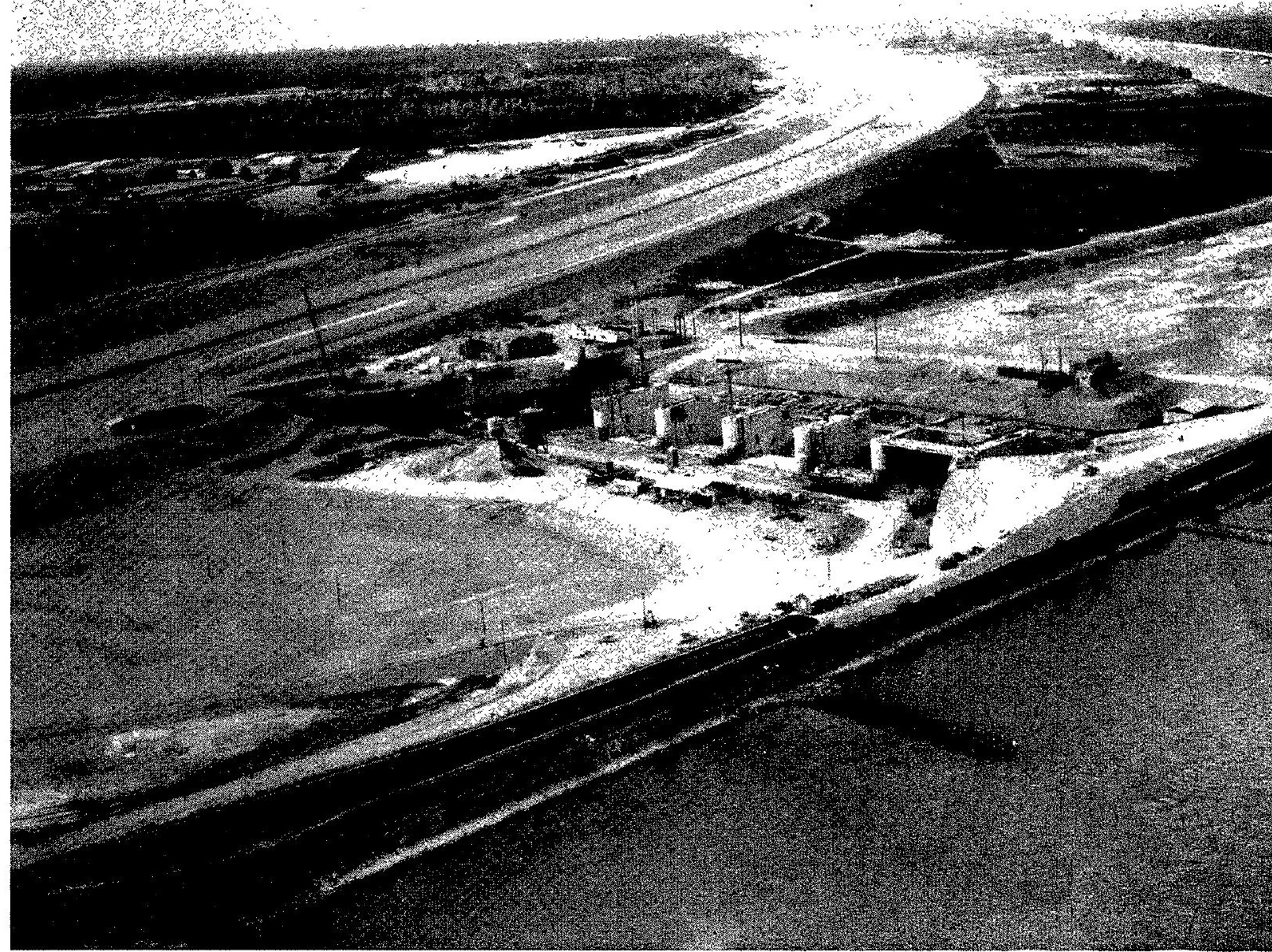

(Photo Baranger, Paris.)

(Photo Baranger, Paris.)

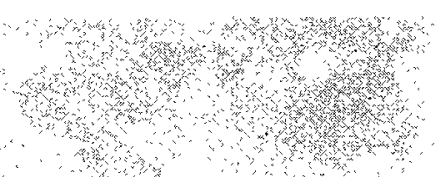

5.

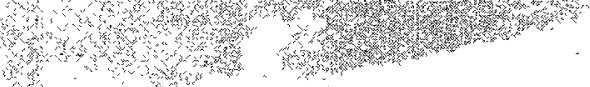

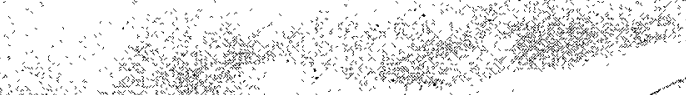

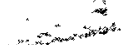

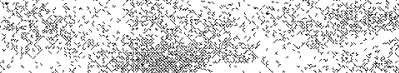
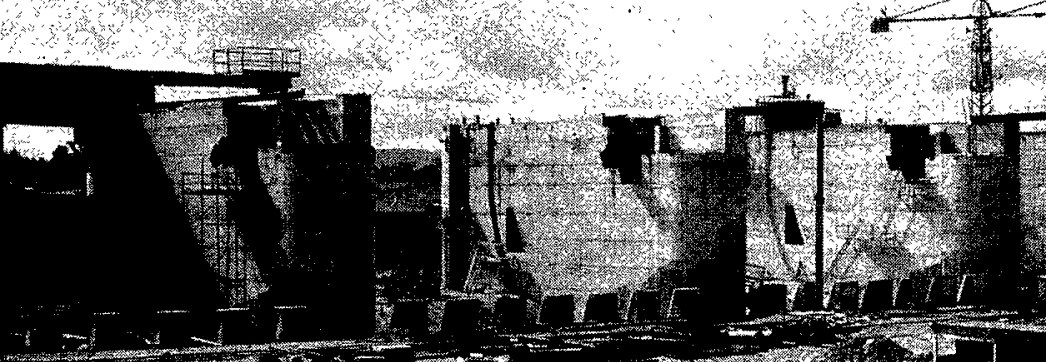

H.

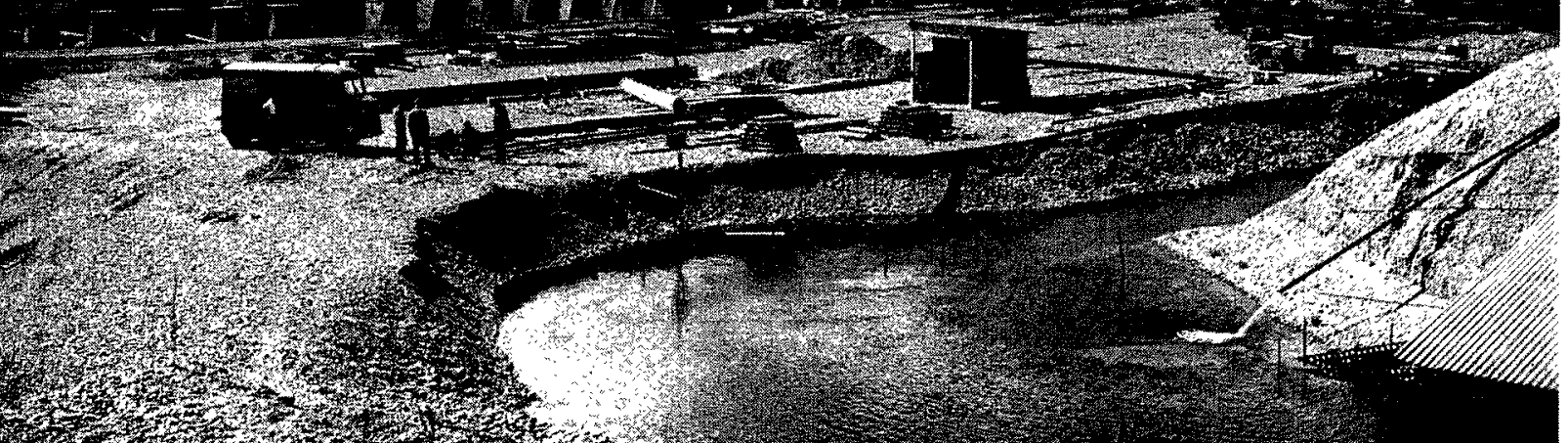

Fia. 10

Barrage de Rhinau.

Vue depuis l'aval sur

les piles $2,3,4,5$ et 6 en construction. 


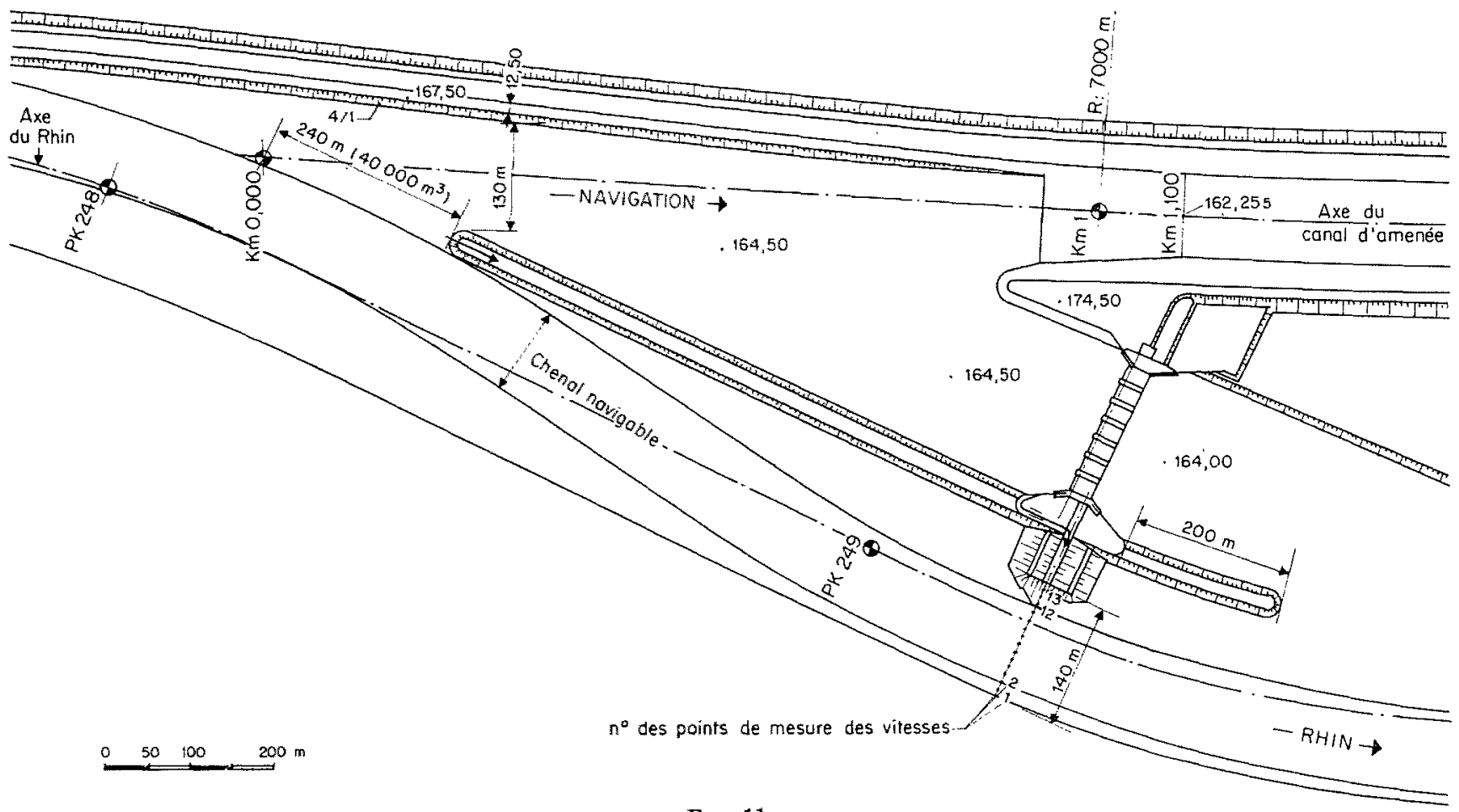

Fia. 11

Rhinau.

Plan général du barrage et de la prise. Situation au jour $\ll J+40 »$.

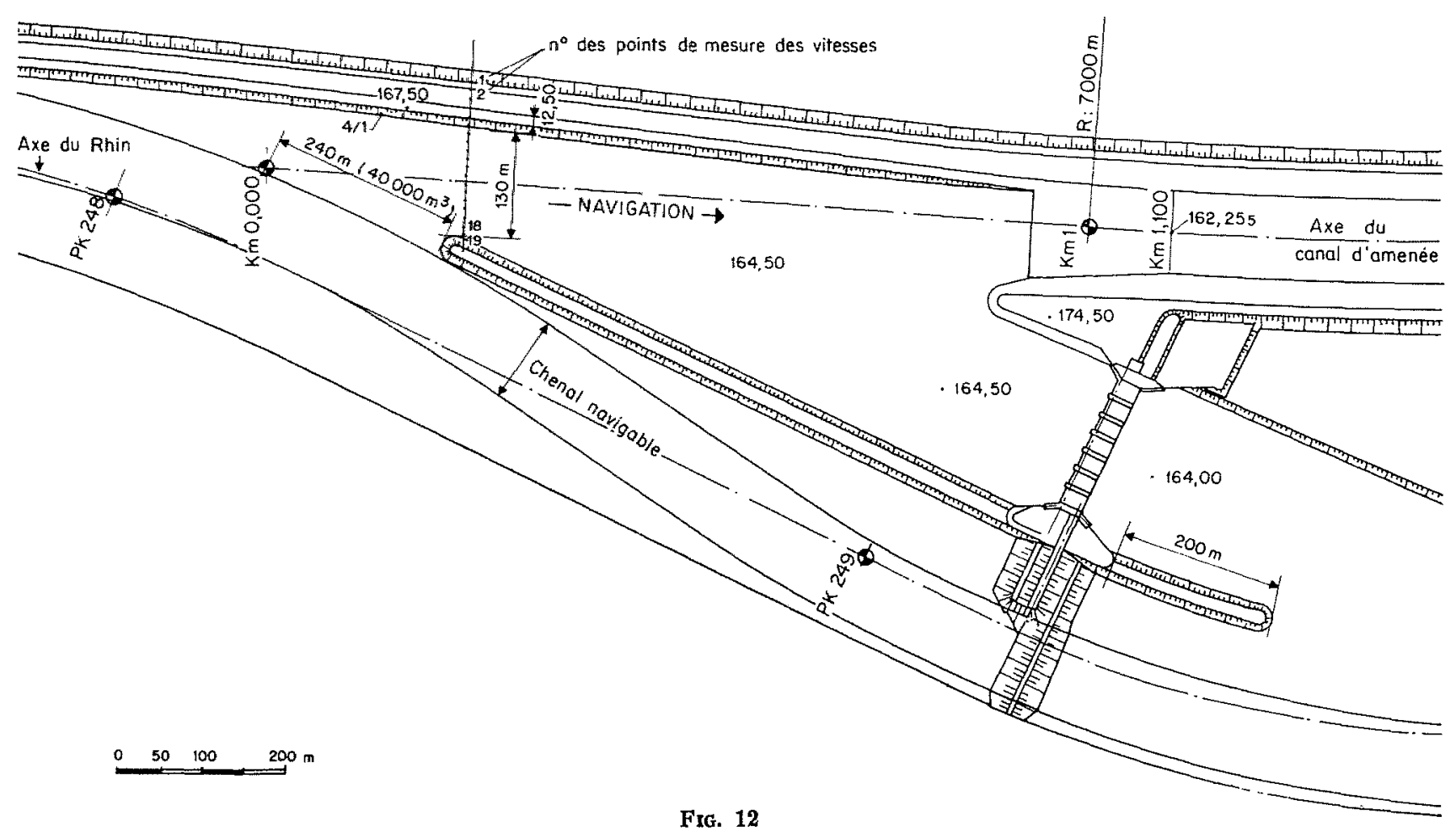

Fic. 12

Rhinau.

Plan général du barrage et de la prise. Situation au jour $\& J+48 》$. 


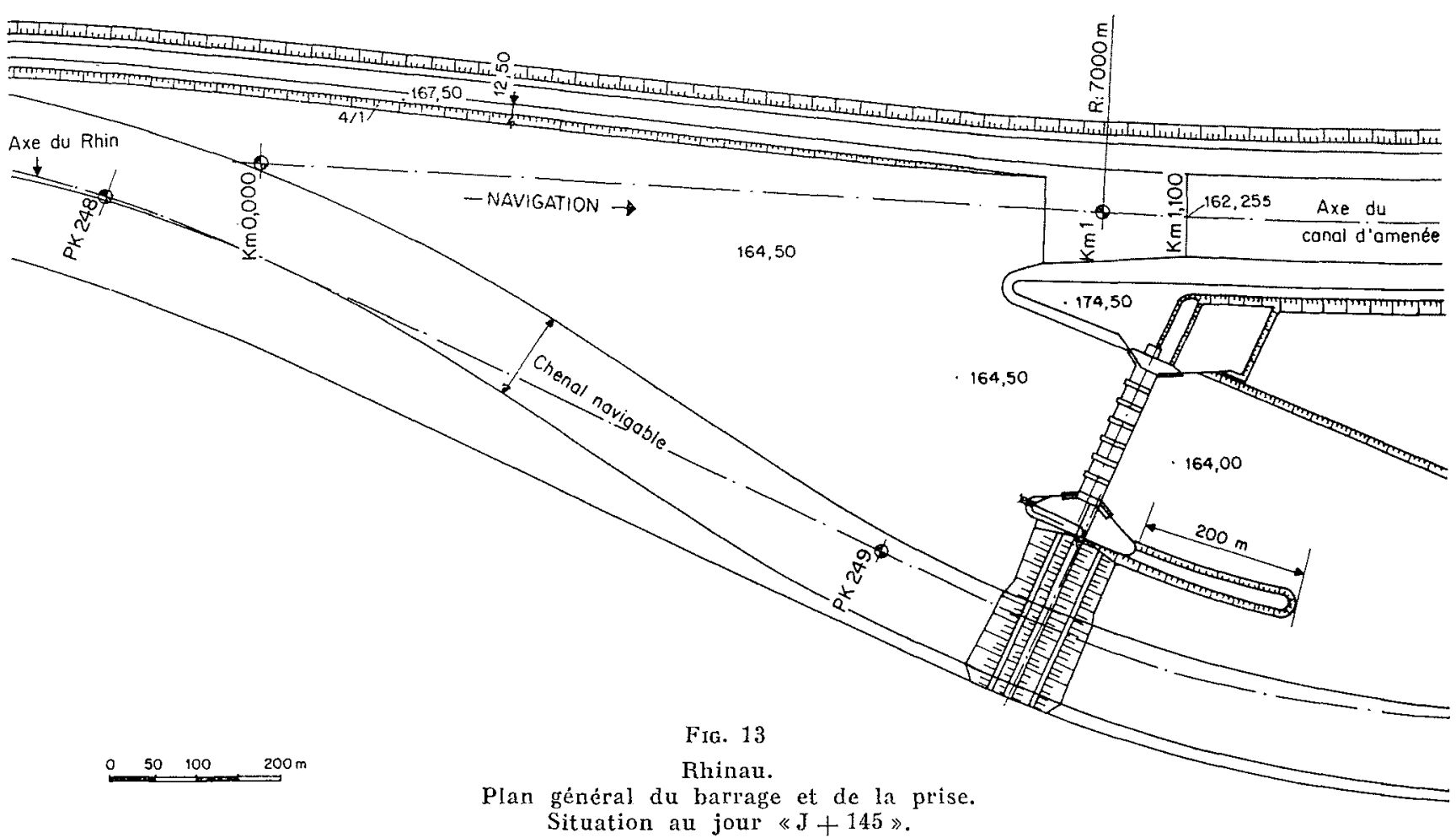

\section{II. - OUVRAGES DE NAVIGATION}

\section{A - Dispositions générales.}

Les ouvrages de navigation de l'aménagement du Rhin ont été fixés par la Commission Centrale pour la Navigation du Rhin et comportent :

$1^{\circ}$ Un ensemble de deux écluses jumelées ayant $185 \mathrm{~m}$ de longueur utile, la grande écluse ayant $23 \mathrm{~m}$ de large et la petite $12 \mathrm{~m}$ de large.

Cot ensemble de deux écluses avec leurs musoirs constitue un ouvrage en maçonnerie de $300 \mathrm{~m}$ de longueur, $76 \mathrm{~m}$ de largeur et d'une hauteur de 30 à $25 \mathrm{~m}$ suivant la chute. Une telle construction, qui demande la mise en place de $200000 \mathrm{~m}^{3}$ de béton et de 1500 à $2000 \mathrm{t}$ d'armatures, justifie une installation de chantier spéciale, indépendante de l'usine.

Cette disposition se recommande par ses fondations dans les alluvions qui nécessitent un rabattement spécial des fouilles par une ou plusieurs stations de pompage flottantes.

$2^{\circ}$ Ces écluses comportent, à l'amont et à l'aval, des garages aux dimensions suivantes:

— Garage amont...... $1000 \mathrm{~m}$ de longueur

$\begin{array}{ccc} & \ldots \ldots & 75 \mathrm{~m} \text { de largeur } \\ \text { - Garage } & \text { aval....... } & 600 \mathrm{~m} \text { de longueur } \\ » & \ldots \ldots & 100 \mathrm{~m} \text { de largeur }\end{array}$

Garages et écluses s'étendent donc sur près de
$2 \mathrm{~km}$ de voie navigable. Compte tenu de la nature alluvionnaire des fondations, le garage amont, dont le plafond est au niveau du sol naturel, est séparé du canal d'amenée qu'alimente l'usine par une digue de 10 à $15 \mathrm{~m}$ de largeur, tandis que le garage aval est creusé dans les alluvions et ne débouche dans le canal de fuite qu'à quelque $450 \mathrm{~m}$ à l'aval de l'usine.

Ces dispositions permettent à la navigation d'entrer et de sortir facilement dans les garages amont et aval sans être gênée par les courants traversiers.

\section{B - Alimentation et vidange des sas des écluses.}

L'alimentation et la vidange des sas a fait l'objet d'une étude approfondie sur modele réduit chez Neyrpic pour la réalisation des écluses d'Ottmarsheim.

L'exploitation des écluses ayant donné entière satisfaction, nous avons conservé depuis 1952 les mêmes circuits hydrauliques pour les écluses des biefs suivants. Les perfectionnements apportés ont été dans les types de vannes d'aqueducs, la constitution des portes des sas, les commandes des vannages, etc., mais la disposition des circuits hydrauliques n'a pas changé. 


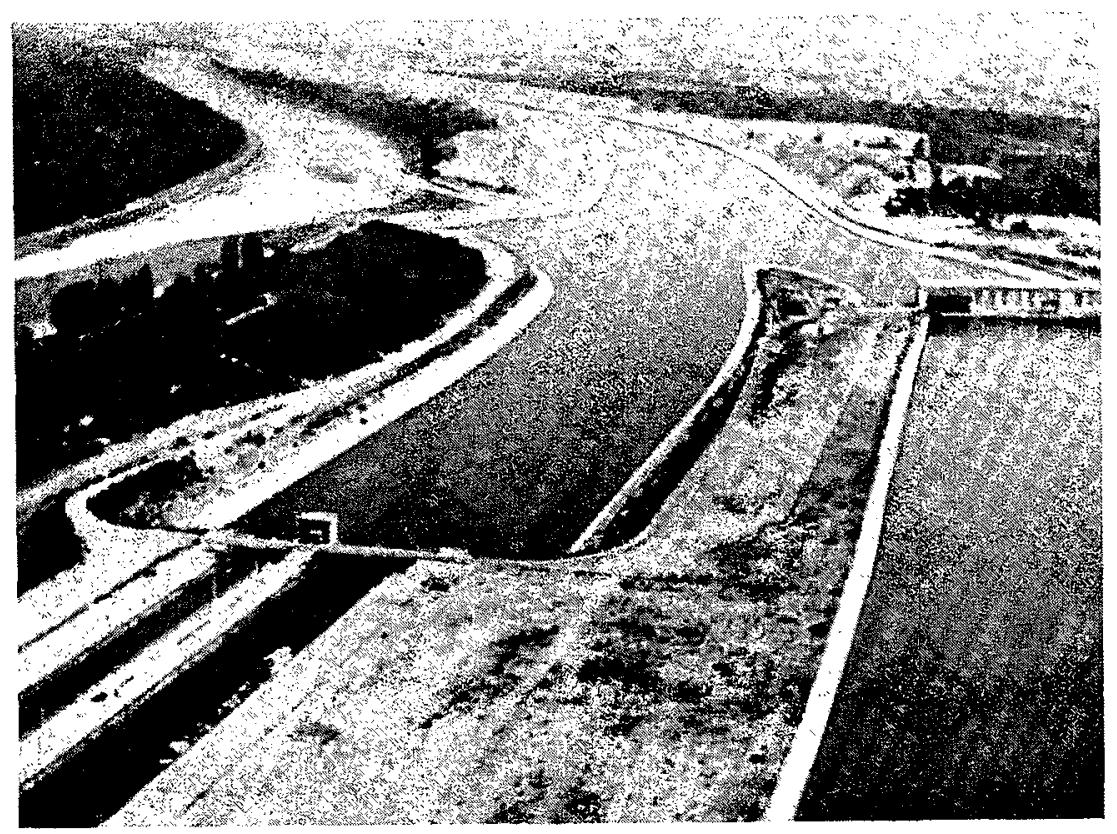

Frg. 14

Fessenheim.

Ouvrages de navigation. (écluses et garages).
Elle perniet :

$1^{\circ}$ une répartition uniforme du débit dans le sas;

$2^{\circ}$ une dispersion de l'énergie dans le sas;

$3^{\prime \prime}$ une alimentation du débit à sa valeur maximale pendant toute la durée, soit du remplissage, soit de la vidange.

Chaque sas est alimente, depuis le garage amont, par deux aqueducs latéraux longitudinaux qui débouchent, l'un au quart, l'autre aux trois quarts de la longueur du sas, dans un aqueduc central placé sous le radier. De part et d'autre de cet aqueduc central, l'eau sort par des orifices rectangulaires dans des chambres de tranquillisation. Ces chambres de tranquillisation sont fermées à leur partie supérieure au niveau du radier du sas par des dalles préfabriquées en béton qui sont perforées pour laissej le passage de l'eau sous forme de jets divisés. La surface de ces orifices est le quintuple de la section des vannes de remplissage. Leurs dimensions sont de $0,40 \mathrm{~m}$ et de $0,31 \mathrm{~m}$ et ceux plus près de l'axe du sas ont reçu une inclinaison pour permettre une plus grande longueur de diffusion.

Pour alimenter le débit à sa valeur maximale pendant toute la durée du remplissage, les vannes des aqueducs sont manœuvrées en trois temps : d'abord à grande vitesse $(50 \mathrm{~mm}$ par seconde) pour atteindre le plus rapidement possible le maximum de remplissage, puis à vitesse lente (28 $\mathrm{mm}$ par seconde) pendant la presque totalite

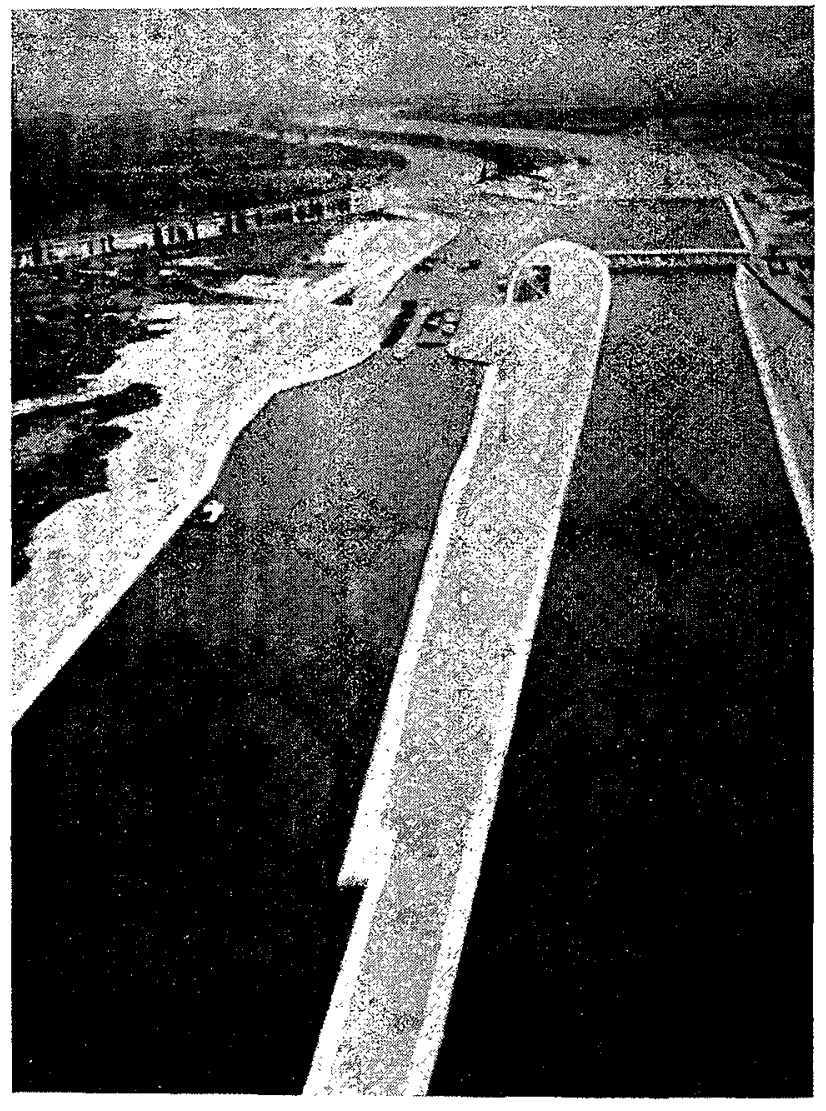

Fig. 15

Fessenheim.

Vue générale du bief à l'aval. 


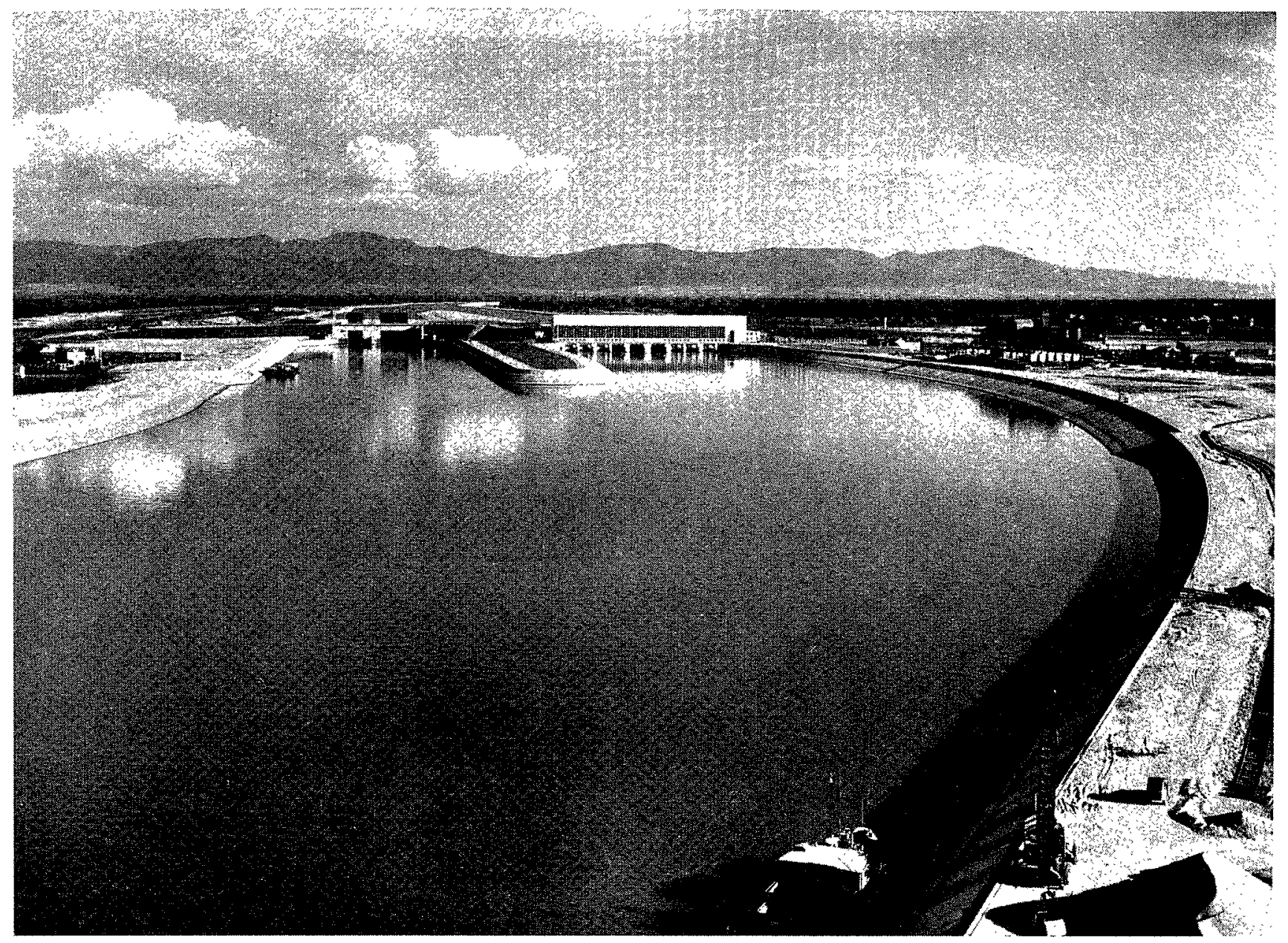

Fig. 26

(Photo Baranger, Paris.)

Volgegrun. Vue générale du garage aval.

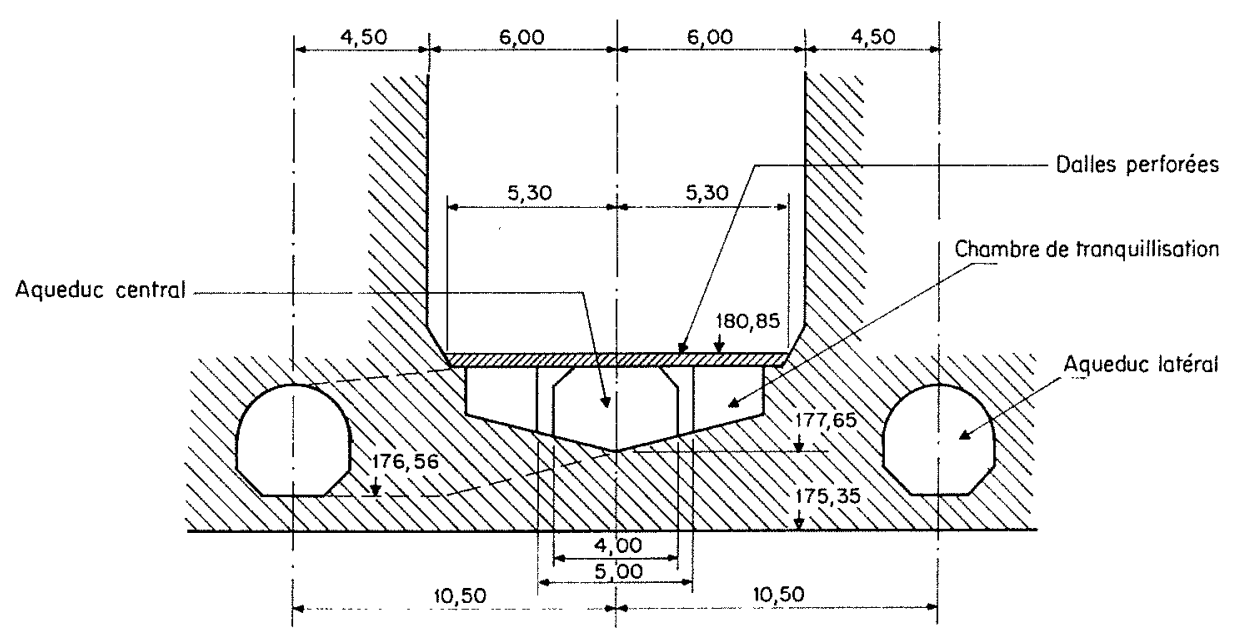

FIG. 17

Vogelgrun. Ecluses.

Coupe en travers, du radier et de l'alimentation. 
du remplissage et enfin à vitesse rapide $(50 \mathrm{~mm}$ par seconde) à la fin du remplissage.

L'entraînement des vannes est réalisé par un ensemble de deux moteurs fonctionnant à vitesses différentes et accouplés par un réducteur. Pour la vitesse rapide, les deux moteurs sont en marche; pour la vitesse lente, seul le petit moteur fonctionne. La vanne étant toujours en mouvement, cette disposition permet un fonctionnement sans vibration, plus simple et plus robuste que celui qui avait été adopté à l'origine à Ottmarsheim, où l'ouverture des vannes se faisait par une succession de mises en fonctionnement el d'arrêts commandés par un commutateur cyclique.

Ce dispositif de commande des vannes per- met en outre de régler facilement le temps de manœuvre en fonction de la hauteur de chute de l'écluse. Avec un débit de $57,5 \mathrm{~m}^{3}$ par seconde par aqueduc, c'est-à-dire de $115 \mathrm{~m}^{3}$ pour l'ensemble des deux aqueducs, la vitesse de montée dans le petit sas est de $3 \mathrm{~m} / \mathrm{mn}$ et dans le grand sas de $1,50 \mathrm{~m} / \mathrm{mm}$.

Les deux sas comportent, depuis Fessenheim, des bollards flottants.

En conclusion, pour ces écluses, nous constatons une nouvelle fois la parfaite concordance entre le modèle et la nature. Les circuits d'eau et les radiers peuvent paraître compliqués et de réalisation délicate; le résultat atteint justifie leur conception pour des écluses importantes de plus de $10 \mathrm{~m}$ de chute.

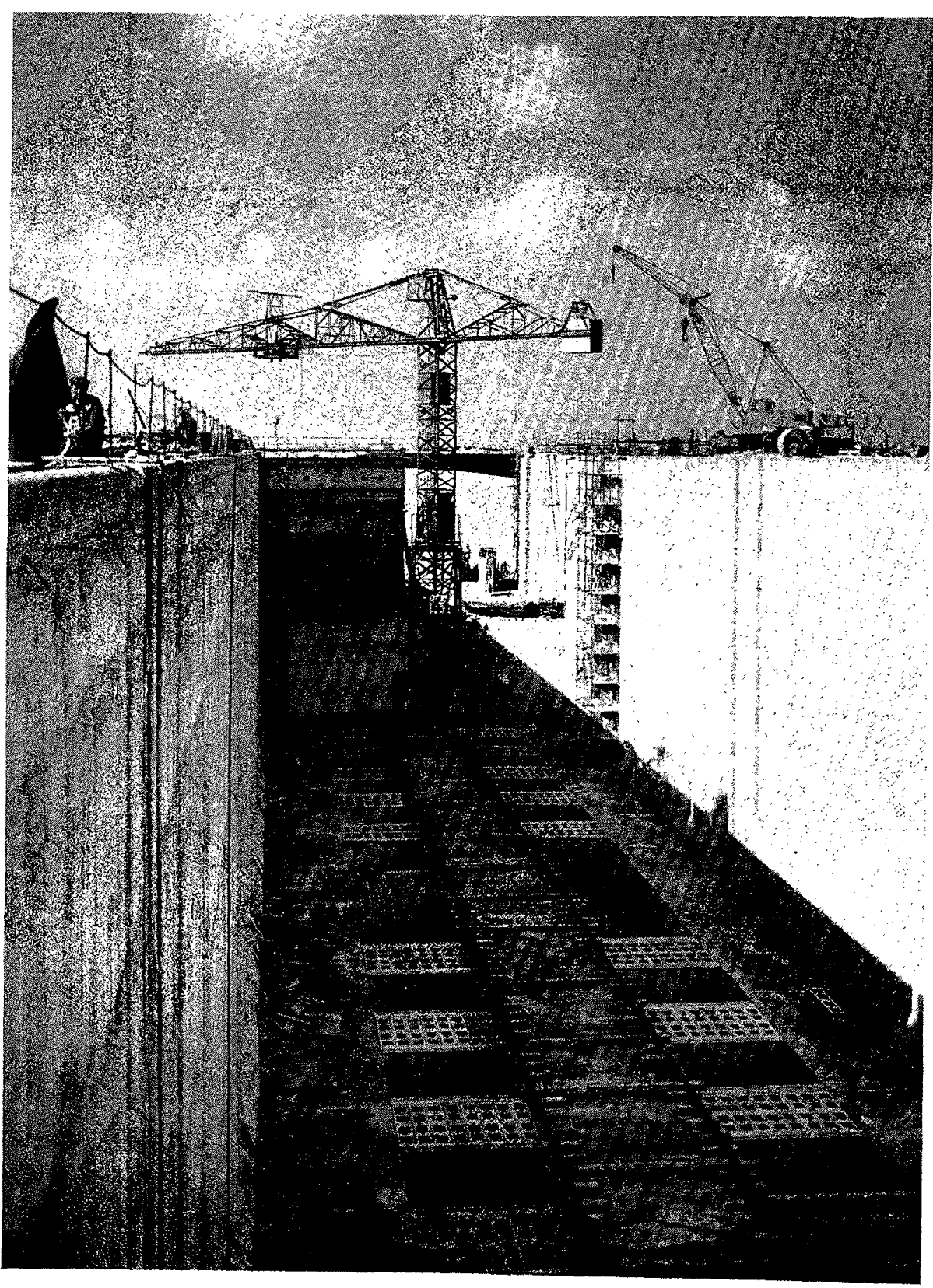

Frg. 18

Ecluses. Photo d'un sas. (Pholo Baranger, Paris.) 


\section{III. - BLOC USINE-DÉCHARGEUR}

\section{A - Protection des fouilles des usines.}

Les blocs usine-déchargeur des usines du Rhin sont fondés, depuis Fessenheim, sur la masse alluvionnaire très profonde $d u$ sillon alsacien.

Les fondations de ces ouvrages, situées à 24 et $26 \mathrm{~m}$ au-dessons du niveau de la nappe phréatique ont nécessité, pour la protection des fouilles, Ia réalisation de cuvettes étanches dans les alluvions après un premier rabattement de surface de 8 à $10 \mathrm{~m}$.

Ces travaux ont fait l'objet de communications au Congrès des Grands Barrages depuis 1955 et on pourrait utilement s'y reporter. J'en rappellerai ici les principes, car ils intéressent un problème d'hydraulique souterraine créé par la présence d'une nappe phréatique particulièrement puissante. Il s'agit, en fait, de travaux considérables, puisque ces cuvettes ont atteint les dimensions suivantes:

$$
\begin{gathered}
\text { - } 175 \text { à } 185 \mathrm{~m} \text { de longueur, } \\
\text { - } 88 \text { à } 116 \mathrm{~m} \text { de largeur, } \\
32 \mathrm{~m} \text { de profondeur. }
\end{gathered}
$$

Leur coût a été pour chaque usine de l'ordre du milliard d'anciens francs et cette dépense s'est révélée rentable par l'absence totale d'aléas au cours de la construction des usines et par des ćconomies substantielles de pompage.

A Fessenheim, les parois verticales de la cuvette ont été réalisées par injection des alluvions avec un coulis d'un mélange d'argile locale et d'argile de Provins et de $20 \%$ de ciment et, pour le fond de la cuvette, par un coulis uniquement de mélange d'argiles sur une épaisseur de $8 \mathrm{~m}$, réduite à partir de Vogelgrun à $5 \mathrm{~m}$.

A Vogelgrun, où la granulométrie des alluvions était à peu près semblable à celle des alluvions de Fessenheim, on a procédé de la même manière par injection des alluvions.

Pour ces deux usines, les zones sableuses des zones injectées ont été l'objet d'un traitement complémentaire au gel de silice.

Pour l'aménagement suivant de Marckolsheim, plus à l'aval, la granulométrie a diminiué de dimensions et nous avons été en présence de poches de sable plus importantes et difficilement injectables. Heureusement, un nouveau procédé, mis au point par l'entreprise chargée des injections, a permis de réaliser la paroi verticale étanche en construisant, sur une profondeur de $40 \mathrm{~m}$, un mur continu moulé dans le sol. Une sondeuse spéciale, comportant un trépan de forme appropriée à la nature des alluvions rencontrées, a creusé une tranchée de $80 \mathrm{~cm}$ de largeur, sans étais ni coffrages, grâce à une boue de forage à base de bentonite déversée dans la fouille pendant le travail. Une machine à bétonner ast venue ensuite déverser un béton maigre à $150 \mathrm{~kg}$ de ciment dans la fouille ainsi ouverte.

A Marckolsheim, on avait prévu l'utilisation du procédé pour la première fois à la réalisation d'une des quatre parois verticales de la cuvette. Les bons résultats de ce premier essai à grande échelle et la nature sableuse des alluvions rencontrées nous incita à faire une seconde paroi verticale.

A Rhinau, le procédé définitivement bien au point fut pratiqué industriellement à la réalisation des quatre parois verticales, le fond de la cuvette continuant à être constitué par injection d'argile sur une hauteur de $5 \mathrm{~m}$.

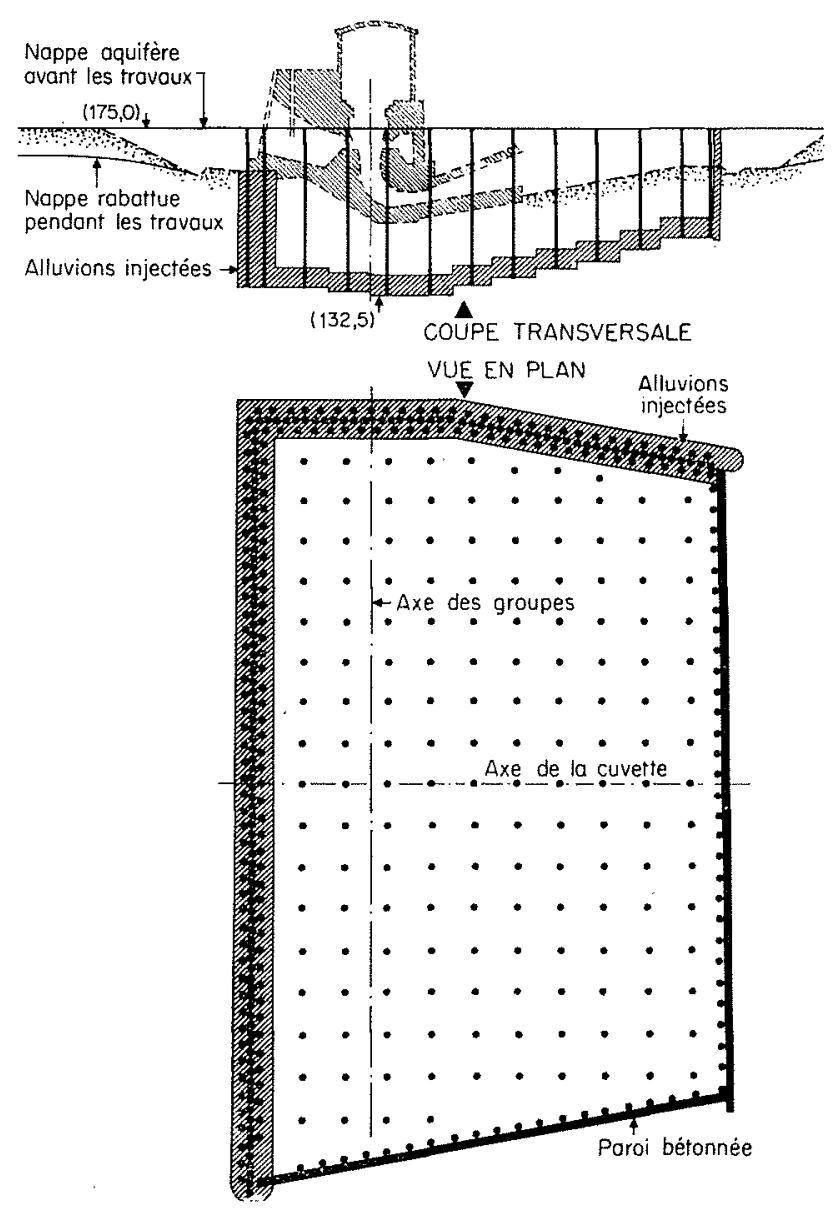

Frg. 19

Cuvette étanche, plan et coupe. 
Ces cuvettes étanches ont donné des résultats très satisfaisants, puisque les débits pompés dans les fouilles ont évolué entre 60 et $200 \mathrm{l} / \mathrm{s}$ à $24 \mathrm{~m}$ sous la nappe, alors que les fouilles des écluses, excavées sans aucune protection à $13 \mathrm{~m}$ sous la nappe, ont nécessité des pompages de 2400 à $3500 \mathrm{I} / \mathrm{s}$.

\section{B - Déchargeurs.}

A partir de Fessenheim, les usines du Rhin ont été construites avec un type de déchargeurs mis au point au Laboratoire de Chatou.

Pour des usines en série sur un canal, le déchargeur a deux fonctions :

- d'une part, il atténue les intumescences, positives à l'amont, négatives à l'aval, provoquées par une interruption brutale de la charge des turbines à la suite d'une coupure volontaire ou involontaire des alternateurs; - d'autre part, il laisse passer le débit dans le canal en cas d'indisponibilité d'un ou de plusieurs groupes.

Les essais faits en mars et mai 1947 sur la relenue de Kembs ont montré que la coupure brusque de la charge totale de l'usine provoquait une intumescence de l'ordre de $80 \mathrm{~cm}$. Cette onde remonte le canal d'amenée. Or, les chalands et les automoteurs ne peuvent supporter que des ondes de l'ordre de $30 \mathrm{~cm}$ sans incident ou avarie. Par ailleurs, le brusque abaissement du niveau dans le garage aval peut provoquer des ruptures d'amarres des bateaux et de violents talonnements des berges, préjudiciables aux bateaux et aux berges.
Les déchargeurs de Kembs et d'Ottmarsheim ont été réalisés par des pertuiș prenant l'eau à l'amont, à la partie supérieure du plan d'eau, pour la restituer à l'aval au-dessus des aspirateurs des turbines sur toute la largeur de l'usine. Ces pertuis étaient fermés à liembs par des vannes-wagons à commande manuelle et relativement lente et à Ottmarsheim par vingt-quatre clapets à servo-moteurs à huile, à commande automatique. L'importance de cette dernière installation, tant au point de vue mécanique que génie civil et sa vitesse de réponse encore lente, nous ont amenés au moment de la conception de Fessenheim à reconsidérer le problème.

Avec la précieuse collaboration du Laboratoire de Chatou et en particulier de M. Brauneau, une série d'études sur modèle réduit a permis de fixer la forme et les dimensions d'un déversoir avec un dispositif de brise-charge, tandis qu'avec Neyrpic se poursuivaient les études de la vanne et d'un servo-moteur à fonctionnement rapide et automatique.

On pent résumer ainsi les caractéristiques de ce déchargeur :

- la prise amont se fait en charge, d'où diminution de la surface à obturer;

-- le brise-charge peut se définir comme un barrage-poids poreux constitué par deux murs minces de profil triangulaire divisant en trois parties la largeur de la passe. Ces murs soutiennent des barreaux horizontaux qui traversent toute la passe et sont scellés de part et d'autre dans les bajoyers. Le haut de cette grille épaisse, constituée de vingt-six bar-

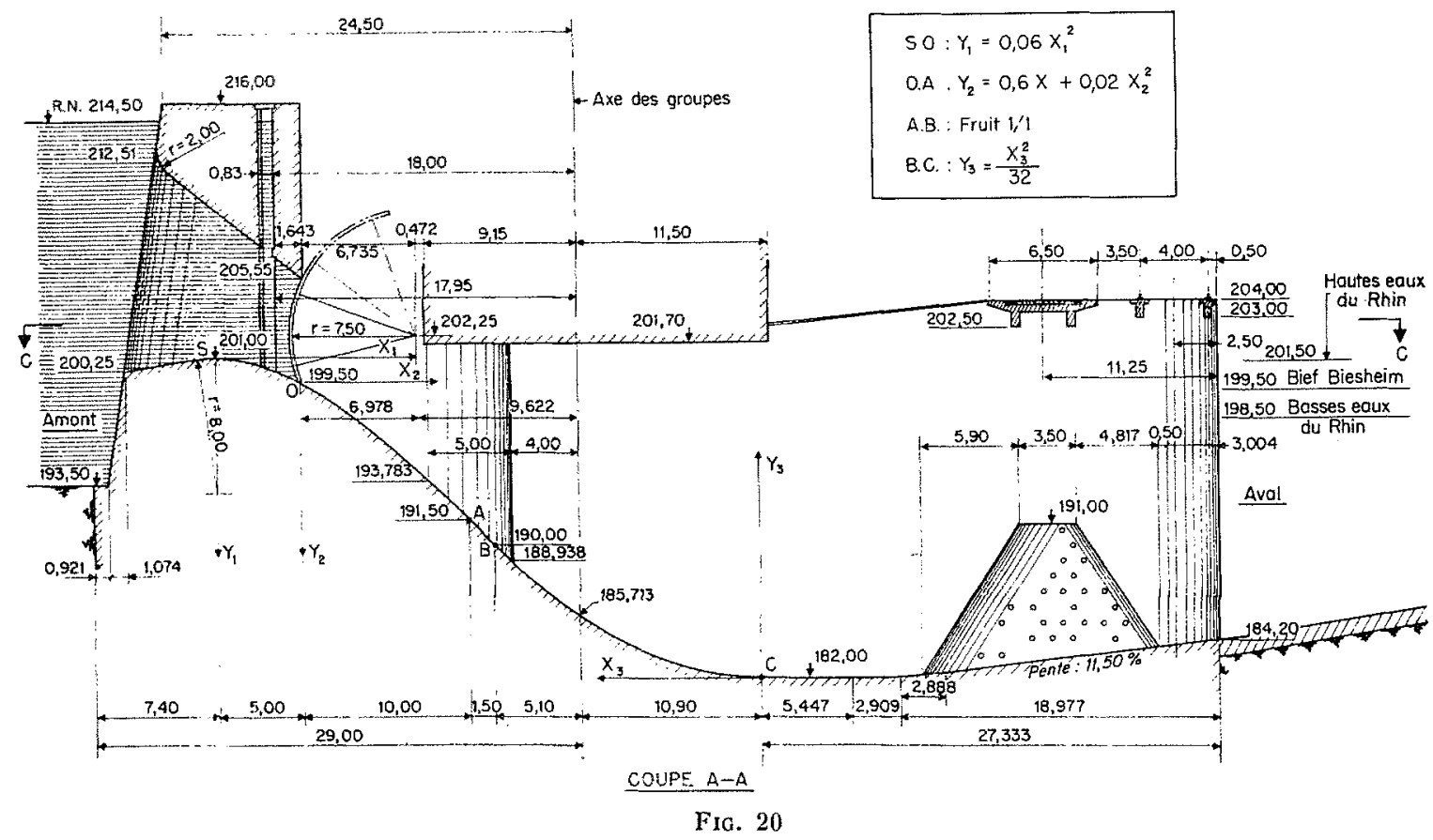

Coupe longitudinale d'un déchargeur. 

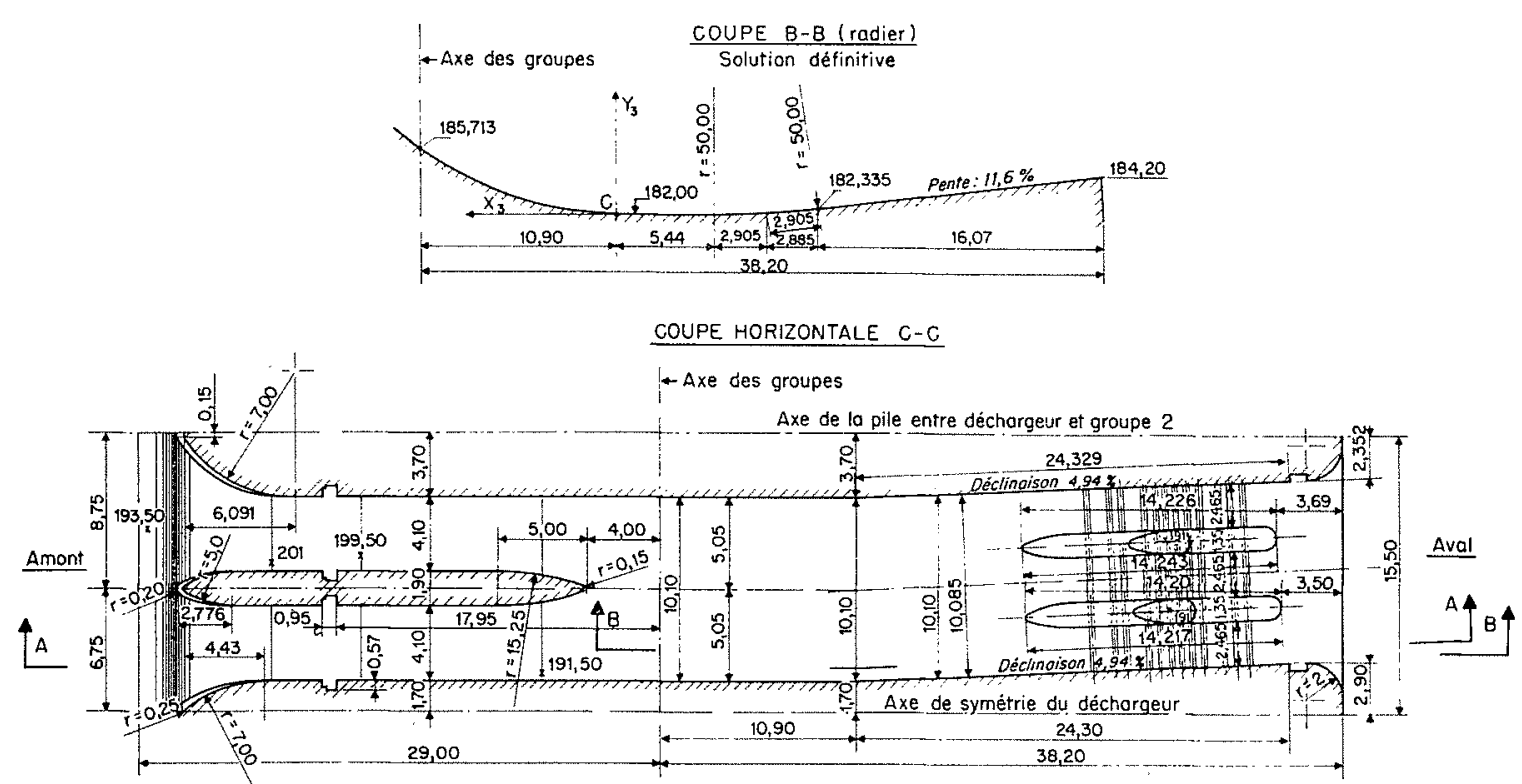

FIG. 21

Plan d'un déchargeur.

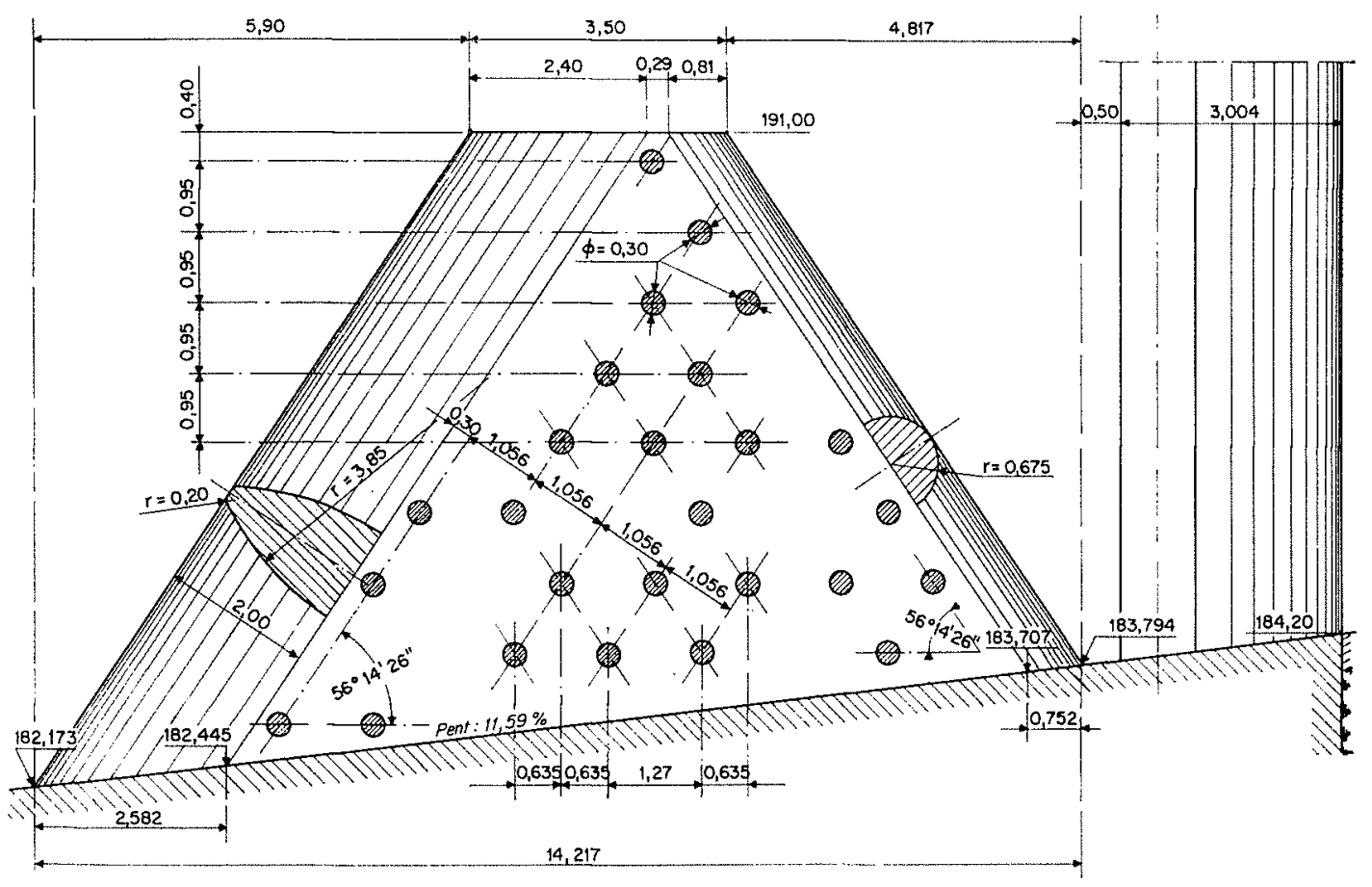

Fig. 22

Brise-charge d'un déchargeur.

reaux de $30 \mathrm{~cm}$ de diamètre, se trouve à 7 ou $8 \mathrm{~m}$ du plan d'eau normal.

Dans un modèle réduit qui permettait, à travers des glaces, de voir les rouleaux horizontaux liés aux ressauts à travers les grilles, l'intuition a joué un grand rôle dans la mise au point du dispositif. On a utilisé le grand nombre de degrés de liberté qu'on avait quant au nombre de barreaux et à leur position et, finalement, on est arrivé à un bon amortissement et à une répartition des vitesses très acceptable à la sortie du pertuis de décharge, la vitesse maximale ne dépassant pas $3,50 \mathrm{~m}$.

Pour éviter toute érosion éventuelle des berges, M. Nizery nous avait conseillé de placer les déchargeurs dans l'axe du canal, de manière à 
épanouir sur toute la largeur les intumescences de surface et de fond. Etant donné la nature alluvionnaire du fond du canal, les essais de Chatou furent complétés par des études sur un modèle à trois dimensions réalisé à Fessenheim cl exploité par le Laboratoire. Ces études permirent de fixer l'angle de sortie des pertuis de décharge et les dimensions des enrochements pour la protection du fond. Pour un fonctionnement des déchargeurs d'une durée illimitée, on trouva que des enrochements de dimensions decroissantes, placés sur une largeur de $60 \mathrm{~m}$ et une Iongueur de $190 \mathrm{~m}$, stabilisaient le fond.

Quant à la commande des rannes des déchargeurs, nous avons demandé à Neyrpic de construire un servo-moteur analogue à celui utilisé pour la fermeture de la turbine et ayant une durée de fonctionnement du même ordre. Ainsi, on a pu compenser l'intumescence par un fonctionnement conjugué et simultané d'une fermeture de la turbine en $4 \mathrm{~s} 1 / 2$ et d'une ouverture du déchargeur en $6 \mathrm{~s}$. L'intumescence a été réduite de 80 à $30 \mathrm{~cm}$.

Remarquons qu'avant d'adopter la disposition du brise-charge, une étude de la vibration des barreaux avait été faite sur un barreau grandeur nature installé dans un pertuis de décharge de lusine de Vaufrey. Il avait été constaté, au moren d'indicateurs de pression, deux genres de vibrations peu influençables par l'encastrement :

1" une fréquence propre de 160 cycles par seconde;

2" une vibration basse frécfuence : 8 cycles par seconde, attribuée aux phénomènes hydraulicpues.

A Fessenheim, après un fonctionnement prolongé en déchargeur avant la mise en service de la totalité des groupes, un barreau s'est des- celle. Aussi, pour les chutes à l'aval, le scellement des barreaux dans les bajoyers a été portó de $0,45 \mathrm{~m}$ à $0,90 \mathrm{~m}$.

Dans les aménagements en dérivation tels qu'on les pratique sur le Rhin depuis Marckolsheim, le ròle des déchargeurs est encore double : d'une part, ils limitent l'importance des intumescences; d'autre part, ils permettent d'assurer l'écoulement d'une partie des crues par le canal de dérivation, les groupes générateurs étant supposés hors service, et d'économiser la construction d'un pertuis du barrage de retenue.

Pour terminer, j'indiquerai que l'installation projetée de groupes Kaplan à axe horizontal, type bulbe, à Gerstheim, permettra d'utiliser ces groupes en déchargeur pour un débit se situant entre 50 et $30 \%$ du débit nominal (naturellement, dans l'hypothèse où il ne serait pas accidenté). Cette possibilité permet de réduire de quatre à trois le nombre des déchargeurs de $275 \mathrm{~m}^{3} / \mathrm{s}$ qui étaient installés depuis Fessenheim.

Cependant, ces groupes -..- de faible inertie ont tendance à s'emballer au moment d'une coupure de Ia charge et d'appeler dans les quatre premières secondes un débit supérieur au débit nominal. Les constructeurs de turbines ne sont pas encore affirmatifs sur lo debit ainsi appelé. Ils citent 50 et $5 \%$.

Les constructeurs devront donner une assurance contractuelle sur le pourcentage et le réduire le plus possible par un fonctionnement convenable du régulateur provocuant la fermeture conjuguée optimale du distributeur el des pales de la roue.

Cette question est importante, car aucun déchargeur ne pourrait compenser une intumescence amont négative. Je la pose, car elle doit être résolue pour éviter toute gène à la navigation.

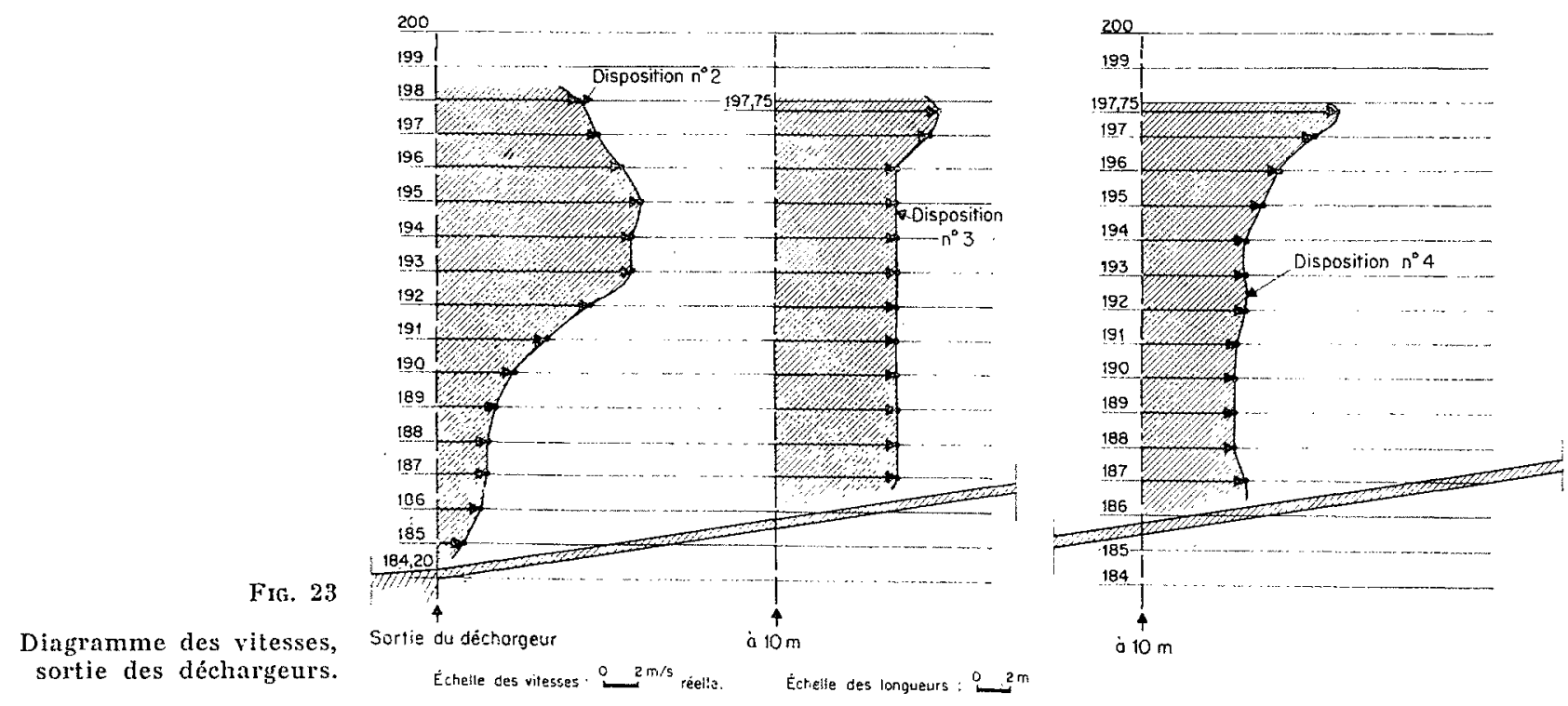




\title{
D I S C US S I O N
}

\author{
Président: M. Durfaut
}

Sur la proposition de M. le Président, l'assemblée, après avoir' entendu l'exposé terminal de M. Gemaehling, avait décidé d'entendre celui de M. Lefoulon, et de procéder ensuite à une discussion commune(1).

M. Ie Président remercie M. Gemaenting et M. Lerooson pour leurs très intéressantes communications et ouvre la discussion.

M. Henry fait quelques remarques en ces termes :

«Les deux très intéressantes communications que nous venons d'entendre ont $m$ is en évidence certaines difïérences entre les méthodes d'aménagement employées sur le Rhône et sur le Rhin, différences qu'explique parfaitement la diversité des conditions.

«Mais le nombre de points communs ou de conclusions communes est beaucoup plus grand que celui des divergences, et parmi ces conclusions communes, je voudrais en citer trois :

1. C'est bien volontiers que je m'associerai à l'hommage que M. Lefovion a rendu aux laboratoires, et je le ferai tont spécialement pour l'aménagement du Rhône, pour lequel les conditions locales nous ont conduits à prévoir une imbrication très accentuée entre les ouvrages de production d'énergie et de navigation (2);

$2^{\circ}$ Ayant exécuté plusieurs barrages en rivière, nous sommes arrivés, nous aussi, à cette conclusion qu'une grande partie de la dépense est consacrée à la construction des batardeaux dans le courant ainsi qu'aux dispositions qu'il faut prendre pour assurer l'écoulement des crues et le passage de la navigation.

\& $\mathrm{Au}$ barrage de Charmes (aménagement de Beauchastel), qui est en cours d'achèvement, nous avons été conduits à implanter trois pertuis sur six hors du lit mincur, ce qui a réduit notablement la part de la dépense consacrée aux batardeaux.

«A Pierre-Bénite, le projet comporte une déviation préliminaire du Rhône pour l'évacuation des crues, et le barrage de retenue, en cours de construction, est établi sur cette déviation, done complètement en dehors du lit mineur.

"Enfin, pour le barrage d'Arras faisant partie de la chute de Saint-Vallier, nous prévoyons que l'ouvrage sera éventuellement hors du lit mineur actuel, avec cette difficulté que ce barrage sera construit sur la rive droite du fleuve, alor's que la prise d'eau se fera sur la rive gauche.

«A Rhinau et à Charmes, le barrage est décalé, par rapport au lit mineur, vers la rive dans laquelle la prise est aménagée.

$3^{\circ}$ A Pierre-Bénite, nous avons à fonder l'usine ef l'écluse sur un sable mollassique qui est actuellement recouvert par $20 \mathrm{~m}$ de gravier rempli d'eau et qui présente une perméabilité voisine $\mathrm{du} \mathrm{cm} / \mathrm{s}$. Ces conditions sont analogues à celles de certaines chutes du Rhin, et nous avons constaté que, si on cherchait à mettre la fouille à sec par épuisements, il faudrait envisager des débits

(1) Voir à ce sujet le compte rendu du Comité Technique page 374 du présent numéro.

(2) Imbrication qui, de toute évidence, complique l'étude hydraulique. d'exhaure énormes $\left(5 \mathrm{~m}^{3} / \mathrm{s}\right.$ en temps normal et $9 \mathrm{~m} \mathrm{~m}^{3} / \mathrm{s}$ avant inondation de la fouille). On aurait eu a faire face à de grosses difficultés de tenue des talus et enfin ces épuisements exceptionnels auraient eu des incidences fâcheuses sur des stations de pompage voisines. C'est ainsi que nous avons été conduits à employer, pour la protection de la fouille contre les infiltrations, le procédé de la paroi moulée dans le sol dont jes travaux du Rhin constituent, en France, la première application à grande échelle.

«A ma connaissance, il n'existe pas de theorie bien complète expliquant la tenue de la tranchée à parois verticales dans laquelle on doit couler le béton d'argile. Je pense que cette tenue est en partie assure par la pression de courant de l'eau contenue dans la tranchée, qui tend à s'écouler dans la mappe en laissant sur les parois de la tranchée un dépôt de bentonite, et il est de fait que dans les applications antérieures du procédé (usine du Rhin et métro de Milan), l'eau lourde était maintenue dans la tranché à un nivea très supérieur à celui de la nappe.

« A Pierre-Bénite, où la paroi mouléc est en cours d'exécution, le niveau de l'eau lourde est en génćmal supérieur à celui de la nappe. Ces jours derniers, cette situation a tendu à s'inverser du fait d'une petite erue du Rhône et il s'est produit des éboulements dans la tranchée. Je crois donc utile de signaler que ce procédé, d'ailleurs très intéressant, paraît devoir etre employé surtout lorsque le niveau de l'eau lourde dans la tranchée est nettement supérieur à celui de la nappe phréatique, condition qu'il est aisé de réaliser en creusant la tranchée dans une diguctte de hauteur suffsante. $\gg$

Monsieur le Président remercie M. HENny de ces explications.

M. Lefoulon demande si la Compagnie Nationale du Rhône a étudié le problème de l'intumescence provoquce par les groupes bulbe à la suite d'une disjonction générale.

M. Cazenave indique que, pour les grompes de PielreBénite, la C.N.R. s'est préoccupée de cette question, qui est liée d'ailleurs à l'étude de la régulation et des survitesses de déclenchement.

D'après l'étude du constructeur, le surdébit de déclenchement, variable suivant la charge du groupe, ne dépasserait pas 5 à $10 \%$ du débit initial.

L'apparente discordance entre ces résultats et ceux qui sont annoncés par allieurs, vient du fait que l'ćlude de Pierre-Bénite a été faite pas à pas, en tenant compte des caractéristiques néelles des groupes foollines en hélice), et non à l'aide des formules linéarisantes habituellement employées gui donnent, pour ces groupes it faible inertie, des résultats largement erronés. Au surplus, on peut agir très largement sur les « surdébits » et les «survitesses» en employant des lois de fermeture du distributeur non linéaires : rapide au début, la fermeture limite le surdébit et la survitesse; lente a la fin, elle limite les coups de belier.

M. InFovion a proposé de consulter le laboratoire de Chatou pour examiner limportance des ondes pouvant résulter des « surdébits 》 de $50 \%$ indiqués. Nous nous proposons, quant à nous, de consulter le département 
* machines » de Chatou pour faire vérifier qu'il est effectivement possible, par un choix convenable de la loi de fermeture, de n'avoir qu'un surdébit négligeable.

Des essais, à ce sujet, ont été lécemment effectués sur les turbines de Bollène; ces essais montrent que l'emploi de lois de mancuvre spéciales permet, sans danger, d’agir largement sur les variations de débit et de vitesse; il semble possible, en particulier, de choisir la loi de façon à annuler les \& surdébits

M. Lefoulon s'inquiète, toutefois, devant des différences de 5 a $50 \%$ et devant les intumescences que produirait une augmentation de $50 \%$ sur un canal comme celui dont il vient de parler; $700 \mathrm{~m}^{3}$ sur 1400 , cela parait inorme. M. LEFovlon espère que les calculs montreront que l'intumescence ne sera pas de plus de $35 \mathrm{~cm}$. Jusqu'à un certain point, cela poserait peut-ctre le problème des gros groupes bulbe, dont M. Lefoulon est partisan, compte tenu de l'économie qu'ils représentent, mais on ne peut pas se permettre de faire cette affirmation devant la navigation rhénane.

M. le Président dit que M. Cazevave a mis en cause des laboratoires. Les représentants de ces laboratoires ont-ils quelque chose à dìre à ce sujet?

M. MeYer rappelle d'abord qu'à ouverture constante du distributeur et à inclinaison constante des pales, le débit des turbines bulbe est grosso modo proportionnel à leur vitesse de rotation, et que, par ailleurs, les groupes bulbe ont, par leur conception même, un faible $\mathrm{PD}^{2}$. Si, après une disjonction, on ne manouvre pas convenablement les organes de contröle du débit, la vitesse de rotation et par conséquent le débit augmentent donc assez rapidement.

En fait, dans le cas des installations classiques quant a leurs caractéristiques hydrauliques et mécaniques, il n'y a pas de difficultés à choisir un temps de manceuvre ou, d'une façon plus générale, une loi de mancuvre qui rend le surdébit relativement faible. Si cela présentait un réel avantage, on pourrait même concevoir un automate rui annulerait dans tous les cas le surdébit. Un tel automate seraił très compliqué et par consécuent sujet à des défailances. On se contente, en pratique, d'une loi de manourre classique qui donne un certain surdébit, fonction de la chute et du débit au moment de la disjonction, et qui ne dépasse pas au maximum 10 à $15 \%$ du débit disjoncté.

M. Meyer remarque que les lois de fermeture permettant de réduire le surdébit préservent aussi le groupe des survitesses trop élevées, des dépressions importantes à I'aval (dues à la coupure du surdébit et à l'effet " pompe $\gg)$ et de certains modes de fonctionnement transitoires présentant des inconvénients, même s'ils ne durent que peu de temps (vibrations, efforts élevés,...).

M. Meyer rappelle que les turbines bulbe, dont il est question, présentent un certain phénomène d'auto-réglage: le surdébit provoque une diminution de la charge motrice (phénomène inverse du coup de bélier) et, par conséquent, une diminution de l'énergie apportée at rotor et done de la survitesse. Ce phénomène d'auto-réglage pro voque, par conséquent, une certaine limitation du surdẻbit.

L'étude complète du phènomène et la détermination exacte du surdébit sont assez compliquées, mais parfaitement faisables, et les méthodes mises en cuvre donnent une précision très satisfaisante.

M. Lefoulon indique que le problème est résolu si on peut se limiter à $5 \%$ d'augmentation du débit, ce qui n'est pas très différent du surdébit intervenant dans le declenchement d'une Raplan ordinaire $(10 \%)$.

M. LIEBEn pense qu'il faut distinguer deux cas :

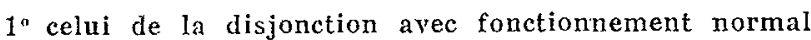
du régulateur;

$2^{\circ}$ celui de la disjonction avec non fonctionnement du régulateur.

Dans le premier cas, il est exact que l'on peut diminuer très sensiblement le surdébit s'écoulant par la turbine en cas de survitesse, en jouant sur la loi de fermeture imposée par le régulateur de vitesse au vannage, et aussi en jouant sur la position des pales de la roue par rapport au vannage au cours de la manouvre.

Il est à remarquer que pour les turbines bulbe, on fait appel à des aubages très rapides dont le débit croît très vite avec la vitesse; ceci n'est pas le cas pour une turbine du type de celles de Blondel, d'autant plus que ces dernières roues sont à six pales. Il semble cependant bien optimiste de penser pouvoir. réduire le surdébit á $5 \%$; une váleur de l'ordre de $15 \%$ paraît plus raisonnable.

Dans le cas de non-réponse du régulateur, il faut s'attendre à des surdébits beaucoup plus considérables, de l'ordre de $50 \%$, car alors on retrouve les caractéristiques propres aux aubages rapides utilisés pour les bulbes.

M. HenRY dit qu'on ne peut avoir des surdébits considérables que sur un seul groupe.

C'est ce que voulait dire M. Mexer qui indique, d'autre part, qu'on peut agir de deux façons sur les Kaplan : on peut en nême temps mouvoir les pales et les vannages et se ménager ainsi une sursécurité car, si le vannage ne ferme pas, on peut agir sur les pales.

L'attaque par les pales est intéressante pour d'autres raisons et l'on est précisément en train d'étudier si on ne peut pas attaquer systématiquement par les pales un certain nombre de Kaplan.

Le Président est heureux de constater, après les difficultés signalées, que l'on finit sur une note légèrement optimiste. 